\title{
Lipotoxicity-Induced mtDNA Release Promotes Diabetic Cardiomyopathy by Activating the cGAS- STING Pathway in Obesity Related Diabetes
}

\section{Xiu Mei Ma}

Macau University of Science and Technology

\section{Kang Geng}

Macau University of Science and Technology

\section{Betty Yuen-Kwan Law}

Macau University of Science and Technology

\section{Peng Wang}

Macau University of Science and Technology

\section{Yue Li Pu}

The Affiliated Hospital of Southwest Medical University

\section{Qing Chen}

The Affiliated Hospital of Southwest Medical University

\section{Hui Wen Xu}

The Affiliated Hospital of Southwest Medical University Department of Transfusion

\section{Xiao Zhen Tan}

The Affiliated Hospital of Southwest Medical University

\section{Zong Zhe Jiang}

The Affiliated Hospital of Southwest Medical University

Yong Xu ( $\nabla$ xywyll@swmu.edu.cn )

The Affiliated Hospital of Southwest Medical University

\section{Research Article}

Keywords: lipotoxicity, mtDNA release, cGAS-STING, diabetic cardiomyopathy

Posted Date: July 27th, 2021

DOl: https://doi.org/10.21203/rs.3.rs-695715/v1

License: (c) (1) This work is licensed under a Creative Commons Attribution 4.0 International License. Read Full License 
Version of Record: A version of this preprint was published at Cell Biology and Toxicology on March 2nd, 2022. See the published version at https://doi.org/10.1007/s10565-021-09692-z. 


\section{Abstract}

Diabetic cardiomyopathy (DCM) is characterized by lipid accumulation, mitochondrial dysfunction, and aseptic inflammatory activation. Mitochondria-derived cytosolic DNA has been reported to induce inflammation by activating cyclic GMP-AMP synthase (cGAS)/the stimulator of interferon genes (STING) pathway in the adipose, liver, and kidney tissue. However, the role of cytosolic mtDNA in the progression of DCM is unclear. In this study, with an obesity-related DCM mouse model established by feeding $\mathrm{db} / \mathrm{db}$ mice with a high-fat diet (HFD), we observed increased mtDNA in the cytosol and activated cGAS-STING signaling pathway during DCM, as well as the downstream targets, IRF3, NF-KB, IL-18, and IL-1 3 . In further study with a palmitic acid (PA)-induced lipotoxic cell model established in $\mathrm{H} 9 \mathrm{C} 2$ cells, we revealed that the cytosolic mtDNA was resulted from PA-induced overproduction of mitochondrial ROS, which also led to the activation of the CGAS/STING system and its downstream targets. Notably, treatment of extracted mtDNA alone was sufficient to activate the cGAS-STING signaling pathway in cultured H9C2 cells. Besides, both knockdown of STING in PA-induced H9C2 cells and inhibition of STING by C-176 injection in the DCM mouse model could remarkably block the inflammation and apoptosis of cardiomyocytes. In conclusion, our study elucidated the critical role of cytosolic mtDNA-induced cGAS-STING activation in the pathogenesis of obesity-related DCM and provided preclinical validation for using a STING inhibitor as a new potential therapeutic strategy for the treatment of DCM.

\section{Introduction}

The International Diabetes Federation estimates that by 2040, nearly 500 million people will be overweight and insulin resistant, and 642 million people will be affected by type 2 diabetes (T2D) [33]. Chronic complications of T2D are the main hazards of diabetes, which often involve the heart, brain, kidney, and other vital organs. Among them, diabetic cardiomyopathy (DCM) is an important cause of heart failure in diabetic patients $[15,39]$. T2D has many harmful effects on the heart, including lipid hhaccumulation, abnormal energy metabolism, oxidative stress, inflammation, apoptosis, changes in fibrosis gene expression, and decreased left ventricular function [35]. In diabetic animal models, the increase of mitochondria-mediated cardiac apoptosis is a major event in DCM development $[4,5]$.

As the energy metabolism center of sugar, fat, and protein, mitochondria account for nearly $30 \%$ of the volume of mature cardiomyocytes. Besides, mitochondria are also the places where intracellular signals integrate and regulate cell homeostasis [31]. Under stress conditions, damaged mitochondria can release some pro-inflammatory signals, such as reactive oxygen species (ROS), in response to changes in the intracellular environment $[6,11]$. In some pathological conditions, such as auto-immune diseases and obesity, increased mitochondrial metabolic stress can lead to excessive ROS production and destruction of mitochondria, which triggers the release of mitochondrial DNA (mtDNA) into the cytoplasm $[3,16]$. In patients with T2D disease, the increase of myocardial triglyceride content is significantly related to the impairment of left ventricular diastolic function [36, 38]. Previous studies have shown that DCM induced by fatty acids is associated with mitochondrial dysfunction, oxidative stress, and inflammation. However, 
the exact molecular mechanism of fatty acids-induced inflammation and cell death in DCM is still unclear.

cGMP-AMP (cGAMP) synthase (cGAS, also known as MB21D1), is considered to be a cytoplasmic DNA biosensor that recognizes DNA from pathogens (bacteria, viruses, etc.). It activates type I interferon response by synthesizing secondary messenger 2'3'-cGAMP in eukaryotic cells in response to the virus and microbial infection $[7,26,30]$. cGAMP and its junction protein interferon gene stimulating protein (STING, also known as TMEM173) binding, promotes the translocation of STING from the endoplasmic reticulum to golgi and forms a complex with tank-binding kinase 1 (TBK1), which is transferred to the internal lysosome where TBK1 phosphorylates transcription factors, including interferon regulatory factor 3 (IRF3) and nuclear factor-kappa B (NF-kB), to initiate signal cascade activation of innate immunityrelated genes, including type I IFN $[10,19,41,48]$. The activation of cGAS-STING protects cells from various pathogens and cancers by enhancing the immune response. However, recent studies have shown that in addition to DNA of microbial origin, the CGAS-STING pathway can also be activated by its own cytoplasmic mtDNA $[23,45]$.

Given that the mitochondrial metabolic stress can lead to mtDNA release and the cGAS-STING system can be activated by cytosolic mtDNA, it is worth noting whether impaired mitochondria contribute the occurrence of DCM through mtDNA-mediated activation of the cGAS-STING pathway. Here, we established an obesity-related DCM mouse model and observed the presence of cytosolic mtDNA, activation of CGAS/STING, and its downstream targets during DCM. Further analysis in palmitic acidinduced lipotoxic cell model showed that PA-induced increase of cytosolic dsDNA and activation of cGAS/STING pathway in a dose-dependent manner. Knockdown of STING in PA-treated H9C2 cells and treatment with STING inhibitor in HFD fed $\mathrm{db} / \mathrm{db}$ mice can respectively block cell death and cardiac dysfunction. Our novel observations suggest that cytosolic mtDNA contributes to DCM through activation of cGAS/STING-mediated inflammatory pathway, indicating that functional inhibition of STING could be a potential therapeutic strategy for DCM patients.

\section{Methods}

\subsection{Materials}

Palmitic acid and N-acetyl-L-cysteine (NAC) were obtained from Sigma (StLouis, MO, USA). Mito-TEMPO was purchased from Santa Cruz Biotechnology (Dallas, TX, USA). STING siRNA and the scrambled siRNA were acquired from RiboBio (Guangzhou, China). Primary antibodies against STING, p65, p-p65, totalIRF3, p-IRF3, IL-1 $\beta$, and Tubulin were purchased from Cell Signaling Technology (Cambridge, UK). Other antibodies against cGAS, dsDNA, GM130, CTGF, and COL1A1 were acquired from Santa Cruz Biotechnology (Dallas, TX, USA). And the anti-mitofilin was purchased from Abcam (Cambridge, UK). The FITC and Cy3 secondary antibodies used in immunofluorescence staining were purchased from Bioss (Beijing, China). In situ cell death detection kit was obtained from Roche. The whole gene DNA extraction 
kit was purchased from FOREGENE (Chengdu, China). The mitochondrial DNA extraction kit was purchased from Biovision (USA). Other chemicals in this study were of analytical grade.

\subsection{Cell culture and treatment}

Cells were cultured as described previously [49]. H9C2 cells, provided by the Institute of Myocardial Electrophysiology, Southwest Medical University, were cultured in DMEM (Hyclone, USA) containing 10\% fetal bovine serum (Sciencell, USA), $100 \mathrm{Imml}^{-1}$ penicillin, and $100 \mathrm{~g} \mathrm{ml}^{-1}$ streptomycin (Beyotime, China) under $5 \% \mathrm{CO}_{2}$ and ambient $\mathrm{O}_{2}$ at $37^{\circ} \mathrm{C}$ (Thermo Scientific, USA).

\subsection{Animals}

Male $\mathrm{db} / \mathrm{db}$ and $\mathrm{db} /+$ mice (4-5 weeks old) were purchased from TengXin (Chongqin, China). All mice were housed in a specific-pathogen-free (SPF) environment (humidity $50 \pm 5 \%$, temperature $20-22^{\circ} \mathrm{C}$ ). $\mathrm{db} / \mathrm{db}$ mice were fed with a $60 \mathrm{Kcal} \%$ fat diet (HFKbio, China) for 8-12 weeks to establish diabetic cardiomyopathy. The bodyweight of mice was measured every week, and fasting blood glucose was measured every two weeks. Mice treated with STING inhibitor were injected intraperitoneally with 750 nmol C-176 (Selleck, USA) per mouse daily in $200 \mu \mathrm{l}$ corn oil (Selleck, USA) for eight weeks. All animal experiment procedures in this study were approved by the National Institutes of Health $(\mathrm{NIH})$ Guide for the Care and Use of Laboratory Animals (Revised 2011) as well as the guidelines of Southwest Medical University (approval number:201903-59).

\subsection{Echocardiography}

Echocardiography was performed as described before [44]. Briefly, M-mode echocardiograms were implemented by a Vevo'3100 ultrasound (VisualSonics, Canada). First, mice were anesthetized with 1.5$2 \%$ isoflurane, and then echocardiography was performed. Cardiac function parameters were collected, including ejection fraction (EF), fractional shortening (FS), Peak E/A ratio et al.

\subsection{Serum triglycerides and inflammatory cytokines assay}

Blood samples in each group were kept at room temperature for 30 minutes, then centrifuged at $3000 \mathrm{~g}$ for 15 minutes $\left(4^{\circ} \mathrm{C}\right)$. After then, the plasma samples were packed in EP tubes and stored at $-80^{\circ} \mathrm{C}$ for the subsequent analyses. The serum triglyceride level was determined by a rapid, convenient and sensitive triglyceride detection kit (Nanjing Jiancheng Bioengineering Research Institute, China). The serum inflammatory cytokines, IL-1 $\beta$ and IL-18, were detected using ELISA kits from Andy Gene (Beijing, China) based on the manufacturer's instructions.

\subsection{Histological analysis}

Hematoxylin-eosin (HE) staining, Tunel, and immunohistochemical staining were performed following the previously described $[22,47]$. Briefly, the myocardial tissues were fixed with $4 \%$ paraformaldehyde, then dehydrated and embedded in paraffin. The hearts were cut into slices with a thickness of $4 \mu \mathrm{m}$ and 
incubated overnight in a thermostat at $37^{\circ} \mathrm{C}$. Then, the slices were put into xylene and a concentration gradient of alcohol for dewaxing. After that, the morphology of cardiomyocytes was observed by $\mathrm{HE}$ staining (Solebo). Besides, cardiomyocyte apoptosis was observed by TUNEL staining (Roche). Furthermore, myocardial fibrosis was evaluated by immunohistochemical staining. After incubated with $3 \% \mathrm{H} 2 \mathrm{O} 2$ and $10 \%$ goat serum for 20 minutes and 1 hour at room temperature respectively, the slices were incubated overnight with anti-CTGF (1:100) and anti-COL1A1 (1:100) at $4{ }^{\circ} \mathrm{C}$, and incubated with antimouse horseradish peroxidase reagent $\left(37^{\circ} \mathrm{C}, 1 \mathrm{~h}\right)$ and $\mathrm{DAB}$ (room temperature, $5 \mathrm{~min}$ ). Finally, the slices were observed with an optical microscope.

\subsection{DNA isolation and mtDNA analysis}

The experiment was carried out as described previously [3]. Briefly, the cultured cardiomyocytes and the freshly purified mouse cardiac tissue were divided into two equal volumes. Whole-cell genomic DNA was extracted by centrifugation column using a DNA extraction kit (FOREGENE). The other used a mitochondrial DNA (mtDNA) extraction kit (Biovision) to extract and purify mtDNA. Cytoplasm free from nuclear, mitochondrial, and endoplasmic reticulum contamination was obtained by high-speed centrifugation. DNA was then isolated from these pure cytoplasmic components using a QIA Quick nucleotide removal column (Qiagen). Quantitative PCR was performed on both whole-cell extracts and cytosolic fractions using nuclear DNA primers (Tert) and mtDNA primers (Dloop1 to 3, and mtND4), and the cycle threshold (CT) values obtained for mtDNA abundance for whole-cell extracts served as normalization controls for the mtDNA values obtained from the cytosolic fractions. This allowed for effective standardization among samples and controlled any variations in the total amount of mtDNA in samples. Using this method, no nuclear Tert DNA was detected in the cytosolic fractions, indicating nuclear lysis did not occur.

\subsection{Western blot}

Western blot analysis was performed as described before [9]. Total proteins from cells or tissue were lysed using RIPA buffer, and the protein concentrations in the cell lysates were assayed by a protein assay dye reagent concentrate (Bio-Rad, USA). Samples were separated by sodium dodecyl sulfatepolyacrylamide gel electrophoresis (SDS-PAGE) and then transferred to polyvinylidene fluoride (PVDF) membranes (pore size $0.45 \mu \mathrm{m}$ ). After being blocked with $5 \%$ BSA for $1 \mathrm{~h}$, membranes were incubated with primary antibodies including cGAS (1:1000), STING (1:1000), p65 (1:500), p-p65 (1:1000), IRF3 (1:1000), p-IRF3 (1:1000), IL-1 $\beta$ (1:1000) and Tubulin (1:5000) at $4{ }^{\circ} \mathrm{C}$ overnight, after being washed with TBST, the membranes were incubated with secondary antibodies for $1 \mathrm{~h}$ at room temperature. Finally, the protein bands were visualized by ECL from Santa Cruz (Dallas, Texas, USA) and analyzed by Image-J software. Cytosolic proteins were normalized to Tubulin or GAPDH.

\subsection{Real-time PCR}

Real-time PCR was performed as described before [20]. Samples were homogenized in TRIzol (Invitrogen), and total RNA was isolated according to the manufacturer s suggested protocol; $1 \mu \mathrm{g}$ of RNA was used 
for CDNA synthesis (Qiagen). Quantitative PCR reactions were performed using SYBR Green (Applied Biosystems) and quantitated using an Applied Biosystems $7900 \mathrm{HT}$ sequence detection system. Duplicate runs of each sample were normalized to GAPDH to determine relative expression levels.

\subsection{Immunofluorescence staining}

The experiment was carried out as described [14]. Briefly, $\mathrm{H} 9 \mathrm{C} 2$ were grown on coverslips in 6-well plates. After adhesion, cells were fixed in $4 \%$ paraformaldehyde and blocked with $5 \%$ bovine serum albumin (BSA). After being gently washed with PBS, the cells were incubated with the primary antibodies including Mitofilin (1:100), dsDNA (1:100), STING (1:100), GM130 (1:100) and p65 (1:100) overnight at 4 C, and then incubated with secondary antibodies conjugated with Fluorescein isothiocyanate (FITC) or cyanine dye 3 (Cy3) for $1 \mathrm{~h}$ at room temperature. 4 6-diamidino-2-phenylindole (DAPI) was used for nuclear staining. Finally, cells were observed under a confocal microscope (Leica, Germany).

\subsection{Statistical analysis}

Statistical analysis of the data was performed using GraphPad Prism 6. Significance was assessed by performing an unpaired two-tailed T-test as indicated in individual figures. No statistical method was used to predetermine sample size. Quantitative data are presented as mean SEM. Statistical significance was set at $* P<0.05,{ }^{\star *} P<0.01, * \star * P<0.001$.

\section{Results}

\subsection{Diabetic cardiomyopathy occurred in HFD fed db/db mice.}

Given that fatty acid (FA) oxidation accounts for $60 \%$ to $90 \%$ of mitochondrial ATP generation under normal conditions and FA accumulation is characteristic of the diabetic heart, we established an obesity related diabetic mouse model to induce diabetic cardiomyopathy by feeding $\mathrm{db} / \mathrm{db}$ mice with HFD for 3 months. Our data showed that compared with the $\mathrm{db} /+$ mice, the BW and FBG of $\mathrm{db} / \mathrm{db}$ mice fed with HFD were significantly higher (Fig.1A), as well as HbA1c and TG (Fig.1B). In addition, the detection of inflammatory markers showed that the concentrations of IL-1 $\beta$ and IL-18 in plasma of $\mathrm{db} / \mathrm{db}$ mice increased (Fig.1B). H \& E staining showed that compared with $\mathrm{db} /+$ mice, $\mathrm{db} / \mathrm{db}$ mice fed with HFD appeared obvious myocardial hypertrophy, narrowing of left ventricular cavity, myocardial fibrosis and even breakage (Fig.1C). Immunohistochemical staining showed that the CTGF and COL1A1 labeled fibers in the myocardial interstitium of $\mathrm{db} / \mathrm{db}$ mice were significantly increased, suggesting that HFD induced myocardial fibrosis in $\mathrm{db} / \mathrm{db}$ mice (Fig.1D). Electron microscope showed that the myocardial myofilament bundles of $\mathrm{db} /+$ mice were arranged neatly, the Z-line and M-line were clearly visible. By contrast, the myocardium of $\mathrm{db} / \mathrm{db}$ mice was disordered or even broken, the Z- and M-line were blurred (Fig.1E). In addition, more apoptotic cells was observed by TUNEL staining in the myocardial interstitium of $\mathrm{db} / \mathrm{db}$ mice fed with HFD (Fig.1F). Together, our data indicate that diabetic cardiomyopathy occurred in HFD fed $\mathrm{db} / \mathrm{db}$ mice. 


\subsection{Mitochondria were impaired with mtDNA release in cardiomyocytes from HFD fed db/db mice.}

As the cytosolic mtDNA derived from damaged mitochondria is a potential inflammatory mediator, we sought to identify the mitochondrial morphology and mtDNA release in DCM. We firstly performed electron microscopic analysis. In db/+ mice, the structure of myocardial mitochondria was complete, the shape was round or oval, and the mitochondrial cristae were complete, rich, and arranged in parallel. Whereas in $\mathrm{db} / \mathrm{db}$ mice, the arrangement of mitochondria was disordered, swollen, and irregular, and most of the cristae were broken, fused, exfoliated, or even myelinated, and some vacuoles could be seen (Fig.2A). These data confirmed that the mitochondria in cardiomyocytes from DCM were severely impaired. Subsequently, we performed co-immunostaining of Mitofilin, the inner membrane protein, and dsDNA to assess the mtDNA release. As expected, we found that compared with $\mathrm{db} /+$ mice, the signals of Mitofilin in cardiomyocytes of $\mathrm{db} / \mathrm{db}$ mice were significantly decreased. Interestingly, we observed a significant increase in the number of dsDNA in the cytoplasm of cardiomyocytes from $\mathrm{db} / \mathrm{db}$ mice (Fig.2B). To quantify the mtDNA release amount, we separated mitochondria and cytosol from the wholecell for the qRT-PCR experiment. The primer Tert and primer Loop 1-3 were used to detect nuclear DNA and mitochondrial DNA, respectively (Fig.2C). Our results showed that Tert was not detected in the isolated and purified myocardial cytoplasmic DNA (Fig.2D), suggesting that the free dsDNA in the cytoplasm was not the nuclear source, and the cytoplasmic DNA extracted in this study was of high purity, and no obvious nucleolysis occurred. After that, we used primers Loop 1-3 to detect mitochondrial DNA in the isolated and purified cytoplasmic DNA. Consistently, the levels of free Loop1, Loop2, and Loop3 in the cytoplasm of $\mathrm{db} / \mathrm{db}$ mice were significantly higher than those of $\mathrm{db} /+$ mice (Fig.2E), indicating that the free dsDNA in the cytoplasm was mainly derived from mitochondria. Taken together, Mitochondria were impaired with mtDNA release in cardiomyocytes from HFD fed $\mathrm{db} / \mathrm{db}$ mice.

\subsection{The cGAS-STING-IRF3/NF-KB pathway was activated in hearts of HFD-fed db/db mice.}

Given that mitochondrial damage led to mtDNA release into cytoplasm and cGAS is considered to be a cytoplasmic DNA biosensor, we next tested whether the cGAS-STING pathway was activated in hearts from HFD-fed diabetic mice. Expectedly, we found that the expression of cGAS and STING increased significantly in the cardiomyocytes of HFD fed db/db mice (Fig.3A). We also found the cGAS and STING gathered around the nucleus by immunostaining assay (Fig.3B-3C). In addition to activation of the cGAS and STING, the downstream targets, NF- KB and IRF3, was also activated in increased phosphorylated form (Fig.3A, 3D \&3E), as well as the expression of NF- KB/IRF3-regulated IL-1 $\beta$ in the cardiomyocytes of HFD-fed db/db mice (Fig.3A). Likewise, the increased mRNA levels of cGAS and STING in HFD-fed db/db mice were confirmed by RT-PCR (Fig.3F), as well as the IL-1 $\beta$ and IL-18 (Fig. 3G). Taken together, these results suggested that The cGAS-STING-IRF3/NF-KB pathway was activated in hearts of HFD fed db/db mice.

\subsection{PA-induced mitochondrial ROS led to mitochondrial damage and mtDNA release in H9C2 cells.}

To investigate whether the lipotoxicity mediates the activation of the cGAS-STING-IRF3/NF-KB pathway in hearts of HFD fed db/db mice, we next used the $\mathrm{H} 9 \mathrm{C} 2$ cell line treated by palmitate acid as a high fat- 
induced lipotoxic cell model. As shown in Fig. 4A, PA treatment led to an increase of ROS level and mitochondrial damage, which were both reversed by NAC, an inhibitor of ROS, indicating that PA-induced ROS led to mitochondrial damage. To confirm that PA treatment leads to mtDNA release into the cytoplasm, we performed co-immunostaining of mitochondria and dsDNA. As shown in Fig.4B, PA induced increase of cytosolic dsDNA in a dose-dependent manner. Further study by qRT-PCR analysis revealed that the increased cytosolic dsDNA induced by PA was derived from mitochondria (Fig.4C). To investigate the source of ROS in the process of mitochondrial injury induced by $\mathrm{PA}$, we pretreated $\mathrm{H} 9 \mathrm{C} 2$ cells with mitochondrial specific ROS scavenger TEMPO. We found that TEMPO could significantly reduce PA-induced intracellular ROS activation and improve mitochondrial membrane potential (Fig.4D). In addition, we evaluated the leakage of mtDNA by fluorescence confocal analysis of dsDNA, mitochondria, and nucleus in PA-treated $\mathrm{H} 9 \mathrm{C} 2$ cells. The results showed that mtDNA leakage in the cytoplasm of $\mathrm{H} 9 \mathrm{C} 2$ cells treated with PA increased, while TEMPO treatment of $\mathrm{H} 9 \mathrm{C} 2$ cells in advance could significantly reduce mtDNA leakage induced by PA (Fig.4E). In summary, these data showed that PA caused mitochondrial damage and mtDNA leakage mainly by activating mitochondrial ROS.

\subsection{PA-induced activation of the cGAS-STING pathway in H9C2 cells.}

To elucidate the effect of PA-induced mtDNA release, we next evaluated the activation of the cGAS-STING pathway in PA-treated $\mathrm{H} 9 \mathrm{C} 2$ cells. As shown in Figure 5A, PA treatment led to an elavated protein level of cGAS and STING in a dose-dependent manner in H9C2 cells. In addition, the downstream targets, phosphorylated IRF3 and NF- $\mathrm{KB}$, were also activated by PA treatment in a dose-dependent manner, as well as IL-1 $\beta$, which was regulated by IRF3 and NF-KB (Fig. 5A). Given that the function of STING is not only determined by its content but also by its location. We next performed co-immunostaining of STING and Golgi matrix protein 130 (GM130), a Golgi marker. In H9C2 cells without PA treatment, STING was weakly co-located with GM130, while PA treatment induced strong co-localization, which directly indicated the functional activation of STING (Fig.5B). Consistently, the concentration of IL-1 $\beta$ and IL-18 in the supernatant of $\mathrm{H} 9 \mathrm{C} 2$ cells after PA treatment were also increased in a dose-dependent manner (Fig.5C), as well as the mRNA levels of cGAS, STING, IL-1 $\beta$, and IL-18 (Fig.5D). Taken together, these results indicated that activation of the cGAS-STING pathway is involved in PA-induced myocardial inflammation.

\subsection{Extracted mtDNA is sufficient to activate cGAS-STING signaling in H9C2 cells.}

As the cGAS is not a mtDNA specific DNA sensor, other sorts of DNA can also activate it. To confirm that mitochondria-derived mIDNA is able to activate the cGAS-STING pathway, we isolated and purified mtDNA to transfect into H9C2 cells. Then the activation of the cGAS-STING pathway and downstream inflammatory activation level were detected by Western blot and qRT-PCR. As shown in Fig 6A\&6C, cGAS and STING expression was activated after mtDNA transfection, accompanying the increased expression of IL-1 $\beta$ and IL-18. In addition, we performed co-immunostaining of STING and Golgi in PA-treated H9C2 cells to evaluate the activation of STING. The results indicated that STING aggregation to Golgi was significantly increased in mtDNA-transfected $\mathrm{H} 9 \mathrm{C} 2$ cells (Fig 6B), suggesting that STING was functionally 
activated by mtDNA treatment. In summary, these data showed that in PA-induced myocardial inflammation, the released cytoplasmic mtDNA acted as the ligand of the cGAS-STING system.

\subsection{Knockdown of STING blocked the PA-induced inflammation and apoptosis in H9C2 cells.}

Given that STING functions as an effector in the cGAS-STING system, we sought to identify whether the inhibition of STING can reverse the effect of PA treatment in H9C2 cells. We employed siRNA to knockdown the STING mRNA. In H9C2 cells transfected with STING siRNA, the expression of STING protein was significantly decreased (Fig.7A), and the localization of STING in Golgi was significantly decreased (Fig.7B), indicating that the transfection of STING siRNA was effective. As expected, STING knockdown could significantly inhibit the activation of NF- KB and the increase of IL-1 $\beta$ in H9C2 cells treated by PA for 24 hours (Fig.7A,7C). In addition, STING knockdown could also significantly blocked the elevated secretion of IL-1 $\beta$ and IL-18 induced by PA treatment in the supernatant of H9C2 cells (Fig.7D). Moreover, we also observed a significant anti-apoptotic effect of STING knockdown on PA-treated H9C2 cells (Fig.7E). Taken together, these data directly indicated that knockdown of STING blocked the PAinduced inflammation and apoptosis in $\mathrm{H} 9 \mathrm{C} 2$ cells.

\subsection{Inhibition of STING ameliorated diabetic cardiomyopathy in HFD fed db/db mice.}

Since that knockdown of STING blocked the PA-induced inflammation and apoptosis in H9C2 cells, we supposed STING as a potential therapeutic target of DCM. To this end, we used a specific inhibitor of STING, C176, to intraperitoneally inject into HFD fed db/db mice (Fig.8A). As shown in Fig.8B, inhibition of STING can reverse the cardiac dysfunction in $\mathrm{db} / \mathrm{db}$ mice fed with HFD, showing an increase in E/A ratio and a shortening of isovolumic relaxation time (IVRT), suggesting an improvement in diastolic cardiac function. In addition, inhibition of STING could partially improve myocardial hypertrophy induced by HFD, but had no significant effect on myocardial contractile function (Fig.8B). To further study the pathological changes, we performed HE staining to observe the cardiac hypertrophy and immunohistochemistry to observe the myocardial fibrosis. The results showed that HFD feeding induced ventricular hypertrophy and myocardial fibrosis in $\mathrm{db} / \mathrm{db}$ mice, which could be partially reversed by $\mathrm{C} 176$ treatment (Fig.8C). Also, HFD feeding induced the increase of inflammatory cytokine IL-1 $\beta \mathrm{in} \mathrm{db} / \mathrm{db}$ mice, while $\mathrm{C} 176$ treatment reduced the production of IL-1 $\beta$ (Fig.8C), which was also confirmed by Western blot (Fig.8D). Besides, C176 treatment also blocked the HFD feeding induced activation of NF-KB, the downstream target of STING, in db/db mice by inhibition of phosphorylated P65 (Fig.8E). In a word, these results suggest that STING functions as a potential therapeutic target for diabetic cardiomyopathy.

\section{Discussion}

As DCM is an important cause of heart failure in diabetic patients [15, 39], it is critical to identify therapeutic targets to prevent disease progression. Recently, a growing body of evidence has demonstrated that the CGAS-STING system plays a central role in numerous diseases such as obesity, nonalcoholic fatty liver disease (NALFD), and acute kidney injury $[3,25,27]$. In this study, we observed the presence of mitochondrial damage, cytosolic mtDNA, and activation of the cGAS-STING signaling 
pathway in cardiomyocytes from an obesity-related DCM mouse model. Using a PA-induced lipotoxicity cell model, we determined that PA-induced mtROS overproduction resulted in mtDNA release, which subsequently activated the CGAS/STING signaling pathway and its downstream targets, NF-KB and IRF3. The activated NF-KB /IRF3 finally promoted the expression of inflammatory factors, IL-18 and IL-1 $\beta$. Notably, either downregulation of STING in H9C2 cells or STING inhibitor injection to HFD fed db/db mice could block the lipotoxicity-induced inflammation and cell death. These findings suggest that STING is a novel, critical molecule involved in the progression of DCM.(Fig. 9).

In mature cardiomyocytes, mitochondria account for nearly $30 \%$ of the volume. It is well known that mitochondrial dysfunction plays a vital role in the pathological process of diabetic cardiomyopathy [24]. A clinical study has reported that mitochondria in cardiomyocytes of diabetic patients showed fragmentation [29]. Mitofilin, an essential protein involved in mitochondrial inner crest formation, was reported to be down-regulated in the diabetic heart by proteome analysis and transgenic overexpression of mitofilin attenuated diabetes mellitus-associated cardiac and mitochondrial dysfunction [43]. However, the molecular mechanism linking mitochondrial dysfunction and cardiac cell death and inflammation is unclear. Here, we also visually observed the damage of the inner mitochondrial membrane of cardiomyocytes in diabetic mice through electron microscopy [Fig. 2]. Of note, we found decreased mitofilin and increased cytoplasmic mtDNA in $\mathrm{H} 9 \mathrm{C} 2$ cells treated with palmitic acid and myocardial tissue of HFD fed db/db mice [Fig. 2\&4], suggesting that mitochondrial damage characterized by mitofilin decreased resulted in mtDNA leakage into the cytosol. In addition, the DNA sensor system, cGAS-STING signaling was activated in PA-treated $\mathrm{H} 9 \mathrm{C} 2$ cells and diabetic hearts [Fig. 3\&5]. The extracted mtDNA treatment alone was sufficient to activate CGAS-STING and the downstream targets in vitro [Fig. 6]. Together, these results suggested that mitochondria-derived cytosolic DNA acts as a critical linker between mitochondrial dysfunction and the pathogenesis of DCM.

It is well known that excessive mitochondrial ROS causes mitochondrial dysfunction in cardiomyocytes by compromising ATP production and inducing cellular dysfunction $[12,50]$. On the one hand, previous studies have suggested that cardiomyocytes from animal models of T1D, T2D, and diabetic patients show increased ROS and altered mitochondrial morphology, including mitochondrial fragmentation, cristae disruption, and swelling $[13,17]$. On the other hand, mitochondrial fragmentation induced by chronic hyperglycemia can be reversed by a superoxide dismutase (SOD) mimetic antioxidant, suggesting that ROS is causally related to mitochondrial dysfunction and controlling the level of mtROS might represent a therapeutic strategy for the treatment of DCM $[37,46]$. However, the role of hyperlipidemiainduced mtROS in the progression of DCM is unknown. In this study, we observed excessive production of ROS and impaired mitochondria in $\mathrm{H} 9 \mathrm{C} 2$ cells treated by palmitic acid in a dose-dependent manner [Fig. 4A]. Although incubation with ROS scavenger NAC could effectively reduce the formation of ROS and reverse the mitochondrial function [Fig. 4A], it is not sure whether the overproduction of ROS is derived from mitochondria as the ROS is not only produced by mitochondria. Notably, we further observed that the mitochondria-targeted antioxidant, mito-TEMPO, significantly inhibited the PA-induced myocardial ROS production [Fig. 4D], indicating the overproduction of mtROS in cardiomyocytes under hyperlipidemia. Consistently, a recently published study reported that injection of mito-TEMPO for 30 
days reduced cardiomyocyte apoptosis, improved cardiac hypertrophy, and dysfunction in diabetic mice [32]. Furthermore, we discovered that mito-TEMPO could not only block the mtROS overproduction, but also significantly reduce the leakage of mitochondrial DNA, suggesting a mechanism of mtROS-induced cytosolic DNA increase [Fig. 4E]. Taken together, our data revealed an early regulated axis of lipid/mtROS/mtDNA in obesity-related DCM.

As we are known, mtDNA is thought to be similar to bacterial DNA and contains pro-inflammatory, unmethylated $\mathrm{CpG}$ motifs [8]. Previous studies have shown that escaping mtDNA can inflame the heart and even cause heart failure $[18,34]$. In a normal physiological state, escaped mtDNA and damaged mitochondria can be digested and degraded by lysosome- mediated autophagy and mitophagy. Whereas in a variety of disease states, such as blood pressure overload and ischemia-reperfusion injury, excess mtDNA accumulates and activates the TLR9 receptor, resulting in persistently activated inflammation response [34]. In addition, it has been shown that oxidized mitochondrial DNA could directly activate NLRP3 inflammasomes during apoptosis [40]. In this study, we reported that the cGAS-STING system was activated in cardiomyocytes from HFD fed $\mathrm{db} / \mathrm{db}$ mice and PA-treated $\mathrm{H} 9 \mathrm{C} 2$ cells, accompanied by the increase of cytosolic mtDNA [Fig. 2-5]. Moreover, the cGAS-STING pathway could be activated by the extracted mtDNA treatment only in cultured $\mathrm{H} 9 \mathrm{C} 2$ cells [Fig. 6]. Our study identified cGAS-STING, not TLR9 receptor, as another mtDNA sensor to mediate lipotoxicity-induced myocardial dysfunction.

As a DNA sensor system, the cGAS-STING pathway was first discovered as a mediator of type I interferon inflammatory responses in immune cells to defend against viral and bacterial infections [26, 28]. A growing body of evidence has shown that the cGAS-STING pathway was also activated by host DNA, which aberrantly localized in the cytosol, contributing to increased sterile inflammation, insulin resistance, and the development of NAFLD $[15,25]$. Following the activation of STING signaling, TBK1 is recruited and activated via its phosphorylated C-terminal tail (CTT) [48]. The activated TBK1 acts as a scaffold to recruit IRF3, which is then phosphorylated in a TBK1-dependent manner. The phosphorylated IRF3 enters the nucleus and promotes the expression of target genes such as interferon [21, 42]. On the other hand, TBK1 also plays a role as the activator of NF-KB, which could promote not only interferon expression but also a transcription of proinflammatory and chemokine factors [1]. Consistently, our results demonstrated that activation of the cGAS-STING pathway was accompanied by increases of the downstream mediators, IRF3 and p65 (one form of NF-KB), and the downstream inflammatory factors, IL-18 and IL-1 $\beta$ [Fig. 3\&5]. Of note, both knockdown of STING in PA treated H9C2 cells [Fig. 7], and inhibition of STING with C176 injection [Fig. 8] can remarkably ameliorate myocardial inflammation and apoptosis. These data suggest CGAS-STING/IRF3/NF-KB axis acts as a mediator in the progression of DCM.

\section{Conclusion}

our study demonstrated that lipotoxicity-induced mtDNA release led to cardiac cell death and fibrosis by activation of CGAS-STING signaling and subsequent inflammation in the obesity-related DCM mouse model. These findings underline the significance of cGAS/STING signaling as a potential therapeutic 
target in DCM, and the preclinical efficacy of STING inhibition as a new therapeutic strategy for the treatment of DCM.

\section{Abbreviations}

T2D, type 2 diabetes; HDF, high-fat diet; DCM, Diabetic cardiomyopathy; PA, palmitic acid; ROS, reactive oxygen species; cGAS, cyclic GMP-AMP synthase; STING, the stimulator of interferon genes; mtDNA, Mitochondrial DNA; TBK1, tank-binding kinase 1; IRF3, interferon regulatory factor 3; NF-KB, nuclear factor-kappa B; NAC, N-acetyl-L-cysteine; EF, ejection fraction; FS, fractional shortening; IVRT, isovolumic relaxation time; HE, Hematoxylin-eosin; SDS-PAGE, sulfate-polyacrylamide gel electrophoresis; PVDF, polyvinylidene fluoride; FA, fatty acid; GM130, golgi matrix protein 130; NALFD, nonalcoholic fatty liver disease; CTT, C-terminal tail.

\section{Declarations}

\section{Funding}

This work was supported by the National Natural Science Foundation of China (No.81970676), Sichuan Science and Technology Program (NO.2020YFS0456), and Luzhou-Southwest Medical University cooperation project (NO.2018LZXNYD-PT01).

\section{Competing interests}

The authors declare no competing interests.

\section{Availability of data and materials}

The datasets generated during and/or analysed during the current study are available from the corresponding author on reasonable request.

\section{Code availability}

No applicable.

\section{Authors' contributions}

Xiu Mei Ma, Zong Zhe Jiang and Yong Xu designed the study. Xiu Mei Ma and Geng Kang performed most of the experiments. Zong Zhe Jiang and Yong Xu provided technical advice. Betty YK Law provided technical support for the main experiments. Xiao Zhen Tan helped with the confocal laser-scanning microscopic analysis. Hui Wen Xu and Peng Wang assisted in raising mice. Yue Li Pu and Qing Chen offered experimental help. Xiu Mei Ma wrote the manuscript. Yong Xu, Zong Zhe Jiang and Betty YK Law provided conceptual advice, edited and revised the manuscript.

\section{Ethics approval and consent to participate}


All animal experiment procedures in this study were approved by the National Institutes of Health $(\mathrm{NIH})$ Guide for the Care and Use of Laboratory Animals as well as the guidelines of Southwest Medical University (approval number:201903-59).

\section{Consent for publication}

All authors have read the paper and agree that it can be published.

\section{Acknowledgements}

The authors thank anyone who contributed towards the article. Furthermore, we thank Mr. Xiao Ping Gao from Institute of Cardiovascular Medicine, Southwest Medical University for his excellent help regarding the animal heart ultrasonography.

\section{References}

1. Abe T, Barber GN (2014) Cytosolic-DNA-mediated, STING-dependent proinflammatory gene induction necessitates canonical NF-KB activation through TBK1. J Virol 88:5328-41 doi:10.1128/JVI.00037-14

2. Bai J, Cervantes C, He S, He J, Plasko GR, Wen J, Li Z, Yin D, Zhang C, Liu M, Dong LQ, Liu F (2020) Mitochondrial stress-activated CGAS-STING pathway inhibits thermogenic program and contributes to overnutrition-induced obesity in mice. Commun Biol 3:257 doi:10.1038/s42003-020-0986-1

3. Bai J, Cervantes C, Liu J, He S, Zhou H, Zhang B, Cai H, Yin D, Hu D, Li Z, Chen H, Gao X, Wang F, O'Connor JC, Xu Y, Liu M, Dong LQ, Liu F (2017) DsbA-L prevents obesity-induced inflammation and insulin resistance by suppressing the mtDNA release-activated cGAS-cGAMP-STING pathway. Proc Natl Acad Sci U S A 114:12196-12201 doi:10.1073/pnas.1708744114

4. Bhagani H, Nasser SA, Dakroub A, El-Yazbi AF, Eid AA, Kobeissy F, Pintus G, Eid AH (2020) The Mitochondria: A Target of Polyphenols in the Treatment of Diabetic Cardiomyopathy. Int J Mol Sci 21:4962 doi:10.3390/ijms21144962

5. Cai L, Li W, Wang G, Guo L, Jiang Y, Kang YJ (2002) Hyperglycemia-induced apoptosis in mouse myocardium: mitochondrial cytochrome C-mediated caspase-3 activation pathway. Diabetes 51:1938-48 doi:10.2337/diabetes.51.6.1938

6. Chen YR, Zweier JL (2014) Cardiac mitochondria and reactive oxygen species generation. Circ Res 114:524-37 doi:10.1161/CIRCRESAHA.114.300559

7. Cheng Z, Dai T, He X, Zhang Z, Xie F, Wang S, Zhang L, Zhou F (2020) The interactions between cGAS-STING pathway and pathogens. Signal Transduct Target Ther 5:91 doi:10.1038/s41392-0200198-7

8. Collins LV, Hajizadeh S, Holme E, Jonsson IM, Tarkowski A (2004) Endogenously oxidized mitochondrial DNA induces in vivo and in vitro inflammatory responses. $J$ Leukoc Biol 75:995-1000 doi:10.1189/jlb.0703328 
9. Costantino S, Akhmedov A, Melina G, Mohammed SA, Othman A, Ambrosini S, Wijnen WJ, Sada L, Ciavarella GM, Liberale L, Tanner FC, Matter CM, Hornemann T, Volpe M, Mechta-Grigoriou F, Camici GG, Sinatra R, Lüscher TF, Paneni F (2019) Obesity-induced activation of JunD promotes myocardial lipid accumulation and metabolic cardiomyopathy. Eur Heart J 40:997-1008 doi:

10.1093/eurheartj/ehy903

10. Ding C, Song Z, Shen A, Chen T, Zhang A (2020) Small molecules targeting the innate immune cGAS-STING-TBK1 signaling pathway. Acta Pharm Sin B 10:2272-2298 doi:10.1016/j.apsb.2020.03.001

11. Dan Dunn J, Alvarez LA, Zhang X, Soldati T (2015) Reactive oxygen species and mitochondria: A nexus of cellular homeostasis. Redox Biol 6:472-485 doi:10.1016/j.redox.2015.09.005

12. Fauconnier J, Andersson DC, Zhang SJ, Lanner JT, Wibom R, Katz A, Bruton JD, Westerblad H (2007) Effects of palmitate on $\mathrm{Ca}(2+)$ handling in adult control and ob/ob cardiomyocytes: impact of mitochondrial reactive oxygen species. Diabetes 56:1136-42 doi:10.2337/db06-0739

13. Galloway CA, Yoon Y (2015) Mitochondrial dynamics in diabetic cardiomyopathy. Antioxid Redox Signal 22:1545-62 doi:10.1089/ars.2015.6293

14. Haag SM, Gulen MF, Reymond L, Gibelin A, Abrami L, Decout A, Heymann M, van der Goot FG, Turcatti G, Behrendt R, Ablasser A (2018). Targeting STING with covalent small-molecule inhibitors. Nature 559:269-273 doi:10.1038/s41586-018-0287-8

15. Isfort M, Stevens SC, Schaffer S, Jong CJ, Wold LE (2014) Metabolic dysfunction in diabetic cardiomyopathy. Heart Fail Rev 19:35-48 doi:10.1007/s10741-013-9377-8

16. Ishikawa H, Ma Z, Barber GN (2009) STING regulates intracellular DNA-mediated, type I interferondependent innate immunity. Nature 461:788-92 doi:10.1038/nature08476

17. Jarosz J, Ghosh S, Delbridge LM, Petzer A, Hickey AJ, Crampin EJ, Hanssen E, Rajagopal V (2017) Changes in mitochondrial morphology and organization can enhance energy supply from mitochondrial oxidative phosphorylation in diabetic cardiomyopathy. Am J Physiol Cell Physiol 312:C190-C197 doi:10.1152/ajpcell.00298.2016

18. Konstantinidis K, Kitsis RN (2012) Cardiovascular biology: Escaped DNA inflames the heart. Nature 485:179-80 doi:10.1038/485179a

19. Lam E, Stein S, Falck-Pedersen E (2014) Adenovirus detection by the cGAS/STING/TBK1 DNA sensing cascade. J Virol 88:974-81 doi:10.1128/JVI.02702-13

20. Li N, Zhou H, Wu H, Wu Q, Duan M, Deng W, Tang Q (2019) STING-IRF3 contributes to lipopolysaccharide-induced cardiac dysfunction, inflammation, apoptosis and pyroptosis by activating NLRP3. Redox Biol 24:101215 doi: 10.1016/j.redox.2019.101215

21. Li Q, Liu C, Yue R, El-Ashram S, Wang J, He X, Zhao D, Zhou X, Xu L (2019) cGAS/STING/TBK1/IRF3 Signaling Pathway Activates BMDCs Maturation Following Mycobacterium bovis Infection. Int J Mol Sci 20:895 doi: 10.3390/ijms20040895

22. Liu S, Du F, Li X, Wang M, Duan R, Zhang J, Wu Y, Zhang Q (2017) Effects and underlying mechanisms of irisin on the proliferation and apoptosis of pancreatic $\beta$ cells. PLoS One 
12:e0175498 doi:10.1371/journal.pone.0175498

23. Liu S, Feng M, Guan W (2016) Mitochondrial DNA sensing by STING signaling participates in inflammation, cancer and beyond. Int J Cancer 139:736-41 doi:10.1002/ijc.30074

24. López-Armada MJ, Riveiro-Naveira RR, Vaamonde-García C, Valcárcel-Ares MN (2013) Mitochondrial dysfunction and the inflammatory response. Mitochondrion 13:106-18 doi:10.1016/j.mito.2013.01.003

25. Luo X, Li H, Ma L, Zhou J, Guo X, Woo SL, Pei Y, Knight LR, Deveau M, Chen Y, Qian X, Xiao X, Li Q, Chen X, Huo Y, McDaniel K, Francis H, Glaser S, Meng F, Alpini G, Wu C (2018) Expression of STING Is Increased in Liver Tissues From Patients With NAFLD and Promotes Macrophage-Mediated Hepatic Inflammation and Fibrosis in Mice. Gastroenterology 155:1971-1984.e4 doi:10.1053/j.gastro.2018.09.010

26. Ma Z, Damania B (2016) The cGAS-STING Defense Pathway and Its Counteraction by Viruses. Cell Host Microbe 19:150-8 doi:10.1016/j.chom.2016.01.010

27. Maekawa H, Inoue T, Ouchi H, Jao TM, Inoue R, Nishi H, Fujii R, Ishidate F, Tanaka T, Tanaka Y, Hirokawa N, Nangaku M, Inagi R (2019) Mitochondrial Damage Causes Inflammation via cGASSTING Signaling in Acute Kidney Injury. Cell Rep 29:1261-1273.e6 doi:10.1016/j.celrep.2019.09.050

28. Marinho FV, Benmerzoug S, Oliveira SC, Ryffel B, Quesniaux VFJ (2017) The Emerging Roles of STING in Bacterial Infections. Trends Microbiol 25:906-918 doi:10.1016/j.tim.2017.05.008

29. Montaigne D, Marechal X, Coisne A, Debry N, Modine T, Fayad G, Potelle C, El Arid JM, Mouton S, Sebti Y, Duez H, Preau S, Remy-Jouet I, Zerimech F, Koussa M, Richard V, Neviere R, Edme JL, Lefebvre P, Staels B (2014) Myocardial contractile dysfunction is associated with impaired mitochondrial function and dynamics in type 2 diabetic but not in obese patients. Circulation 130:554-64 doi:10.1161/CIRCULATIONAHA.113.008476

30. Morehouse BR, Govande AA, Millman A, Keszei AFA, Lowey B, Ofir G, Shao S, Sorek R, Kranzusch PJ (2020) STING cyclic dinucleotide sensing originated in bacteria. Nature 586:429-433 doi:10.1038/s41586-020-2719-5

31. Mottis A, Herzig S, Auwerx J (2019) Mitocellular communication: Shaping health and disease. Science 366:827-832 doi:10.1126/science.aax3768

32. Ni R, Cao T, Xiong S, Ma J, Fan GC, Lacefield JC, Lu Y, Le Tissier S, Peng T (2016) Therapeutic inhibition of mitochondrial reactive oxygen species with mito-TEMPO reduces diabetic cardiomyopathy. Free Radic Biol Med 90:12-23 doi:10.1016/j.freeradbiomed.2015.11.013

33. Ogurtsova K, Fernandes JD, Huang Y, Linnenkamp U, Guariguata L, Cho NH, Cavan D, Shaw JE, Makaroff LE (2017) IDF Diabetes Atlas: Global estimates for the prevalence of diabetes for 2015 and 2040. Diabetes Res Clin Pract 128:40-50 doi:10.1016/j.diabres.2017.03.024

34. Oka T, Hikoso S, Yamaguchi O, Taneike M, Takeda T, Tamai T, Oyabu J, Murakawa T, Nakayama H, Nishida K, Akira S, Yamamoto A, Komuro I, Otsu K (2012) Mitochondrial DNA that escapes from autophagy causes inflammation and heart failure. Nature 485:251-5 doi:10.1038/nature10992 
35. Peterson LR, Gropler RJ (2020) Metabolic and Molecular Imaging of the Diabetic Cardiomyopathy. Circ Res 126:1628-1645 doi:10.1161/CIRCRESAHA.120.315899

36. Rijzewijk LJ, van der Meer RW, Smit JW, Diamant M, Bax JJ, Hammer S, Romijn JA, de Roos A, Lamb $\mathrm{HJ}$ (2008) Myocardial steatosis is an independent predictor of diastolic dysfunction in type 2 diabetes mellitus. J Am Coll Cardiol 52:1793-9 doi:10.1016/j.jacc.2008.07.062

37. Schilling JD (2015) The mitochondria in diabetic heart failure: from pathogenesis to therapeutic promise. Antioxid Redox Signal 22:1515-26 doi:10.1089/ars.2015.6294

38. Schulze PC, Drosatos K, Goldberg IJ (2016) Lipid Use and Misuse by the Heart. Circ Res 118:1736-51 doi:10.1161/CIRCRESAHA.116.306842

39. Seferović PM, Paulus WJ (2015) Clinical diabetic cardiomyopathy: a two-faced disease with restrictive and dilated phenotypes. Eur Heart J 36:1718-27, 1727a-1727c doi:10.1093/eurheartj/ehv134

40. Shimada K, Crother TR, Karlin J, Dagvadorj J, Chiba N, Chen S, Ramanujan VK, Wolf AJ, Vergnes L, Ojcius DM, Rentsendorj A, Vargas M, Guerrero C, Wang Y, Fitzgerald KA, Underhill DM, Town T, Arditi M (2012) Oxidized mitochondrial DNA activates the NLRP3 inflammasome during apoptosis. Immunity 36:401-14 doi:10.1016/j.immuni.2012.01.009

41. Tanaka Y, Chen ZJ (2012) STING specifies IRF3 phosphorylation by TBK1 in the cytosolic DNA signaling pathway. Sci Signal 5:ra20 doi:10.1126/scisignal.2002521

42. Tanaka Y, Chen ZJ (2012) STING specifies IRF3 phosphorylation by TBK1 in the cytosolic DNA signaling pathway. Sci Signal 5:ra20 doi:10.1126/scisignal.2002521

43. Thapa D, Nichols CE, Lewis SE, Shepherd DL, Jagannathan R, Croston TL, Tveter KJ, Holden AA, Baseler WA, Hollander JM (2015) Transgenic overexpression of mitofilin attenuates diabetes mellitus-associated cardiac and mitochondria dysfunction. J Mol Cell Cardiol 79:212-23 doi:10.1016/j.yjmcc.2014.11.008

44. Wei WY, Ma ZG, Zhang N, Xu SC, Yuan YP, Zeng XF, Tang QZ (2018) Overexpression of CTRP3 protects against sepsis-induced myocardial dysfunction in mice. Mol Cell Endocrinol 476:27-36 doi:10.1016/j.mce.2018.04.006

45. West AP, Shadel GS (2017) Mitochondrial DNA in innate immune responses and inflammatory pathology. Nat Rev Immunol 17:363-375 doi:10.1038/nri.2017.21

46. Westermeier F, Navarro-Marquez M, López-Crisosto C, Bravo-Sagua R, Quiroga C, Bustamante M, Verdejo HE, Zalaquett R, Ibacache M, Parra V, Castro PF, Rothermel BA, Hill JA, Lavandero S (2015) Defective insulin signaling and mitochondrial dynamics in diabetic cardiomyopathy. Biochim Biophys Acta 1853:1113-8 doi:10.1016/j.bbamcr.2015.02.005

47. Xiao Y, Wu QQ, Duan MX, Liu C, Yuan Y, Yang Z, Liao HH, Fan D, Tang QZ (2018) TAX1BP1 overexpression attenuates cardiac dysfunction and remodeling in STZ-induced diabetic cardiomyopathy in mice by regulating autophagy. Biochim Biophys Acta Mol Basis Dis 1864:17281743 doi:10.1016/j.bbadis.2018.02.012 
48. Zhang C, Shang G, Gui X, Zhang X, Bai XC, Chen ZJ (2019) Structural basis of STING binding with and phosphorylation by TBK1. Nature 567:394-398 doi:10.1038/s41586-019-1000-2

49. Zhao M, Lu L, Lei S, Chai H, Wu S, Tang X, Bao Q, Chen L, Wu W, Liu X (2016) Inhibition of Receptor Interacting Protein Kinases Attenuates Cardiomyocyte Hypertrophy Induced by Palmitic Acid. Oxid Med Cell Longev 2016:1451676 doi: 10.1155/2016/1451676

50. Zorov DB, Juhaszova M, Sollott SJ (2014) Mitochondrial reactive oxygen species (ROS) and ROSinduced ROS release. Physiol Rev 94:909-50 doi:10.1152/physrev.00026.2013

\section{Figures}


A
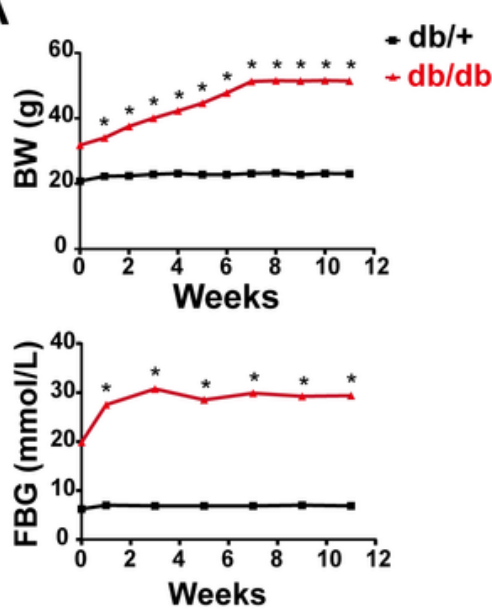

B
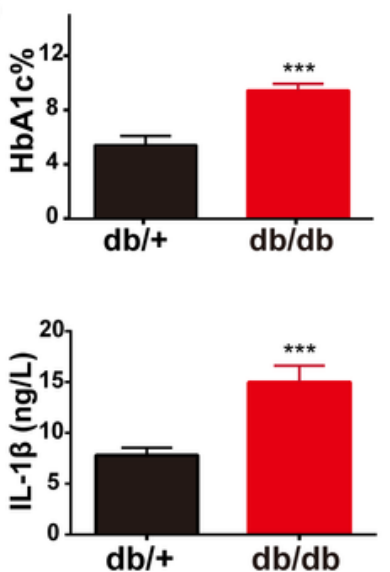
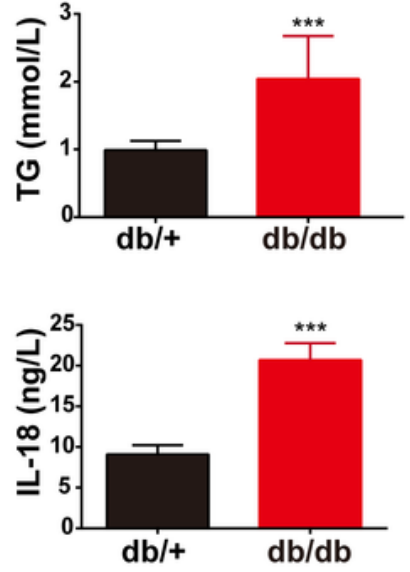

C

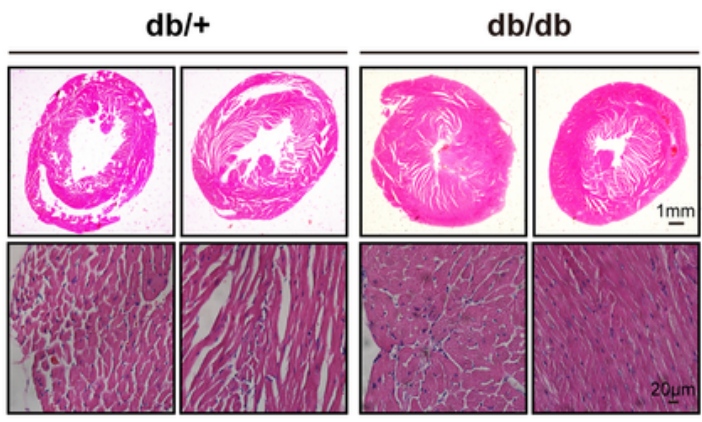

E

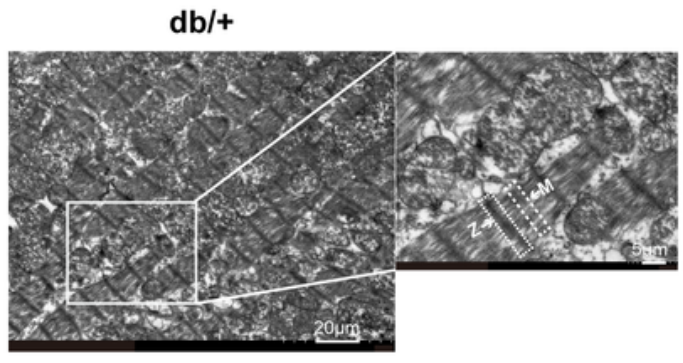

$\mathbf{F}$

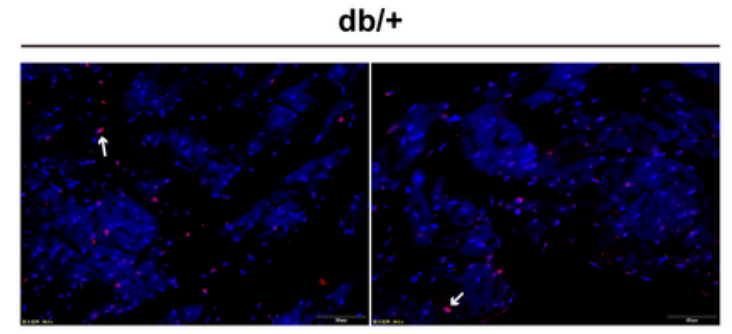

DAPI Tunel
D
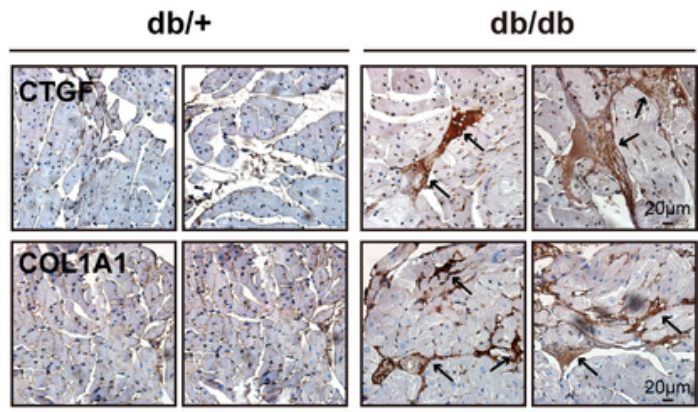

$\mathrm{db} / \mathrm{db}$

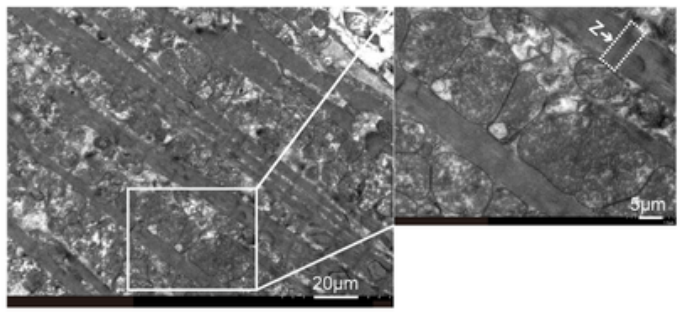

$\mathrm{db} / \mathrm{db}$

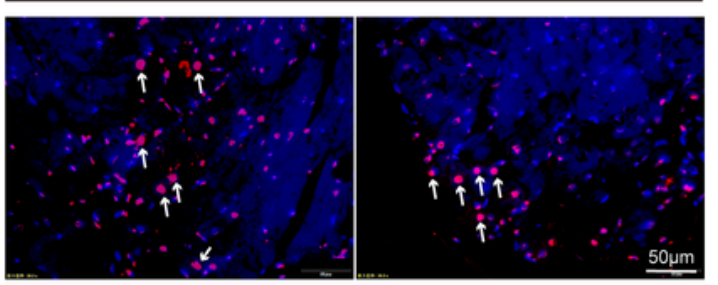

\section{Figure 1}

Diabetic cardiomyopathy occurred in HFD fed db/db mice. A Dynamic changes of body weight (BW) and fasting blood glucose (FBG) in $\mathrm{db} /+$ and $\mathrm{db} / \mathrm{db}$ mice during ND and HFD feeding, respectively $(\mathrm{n}=8$, *P区 0.05 vs 0 week). B The level of HbA1c, TG, IL-1 $\beta$ and IL-18 in the blood of two groups of mice ( $n=4$, ***P区 $0.001 \mathrm{vs} \mathrm{db/+} \mathrm{group).} \mathrm{C} \mathrm{Representative} \mathrm{images} \mathrm{of} \mathrm{the} \mathrm{morphological} \mathrm{analysis} \mathrm{by} \mathrm{H \& E} \mathrm{staining} \mathrm{of} \mathrm{heart}$ tissue. D CTGF and COL1A1 expressions in hearts of HFD-fed db/db mice were visualized by IHC 
staining. E Representative transmission electron microscopy images of myofilament arrangement in $\mathrm{db} /+$ mice and $\mathrm{db} / \mathrm{db}$ mice. The arrow indicated Z-line or M-line. F Representative images of cardiomyocyte apoptosis reflected by TUNEL staining. The arrow indicated apoptotic cell.

A
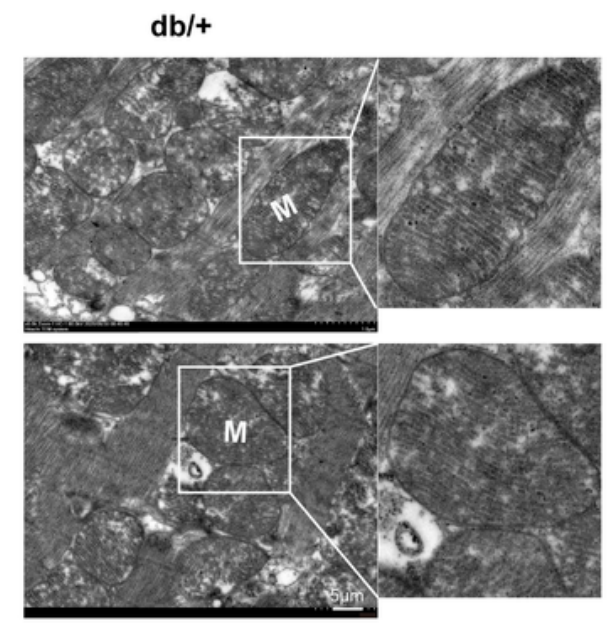

$\mathrm{db} / \mathrm{db}$
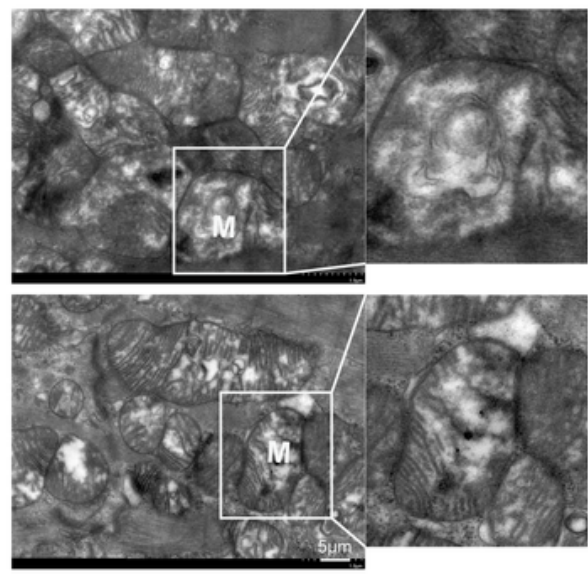

B

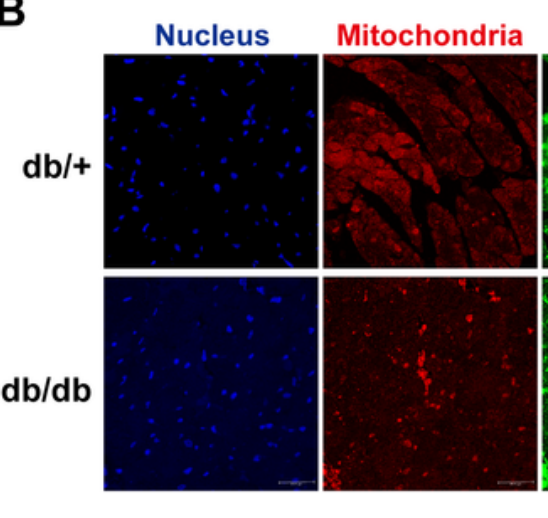

C
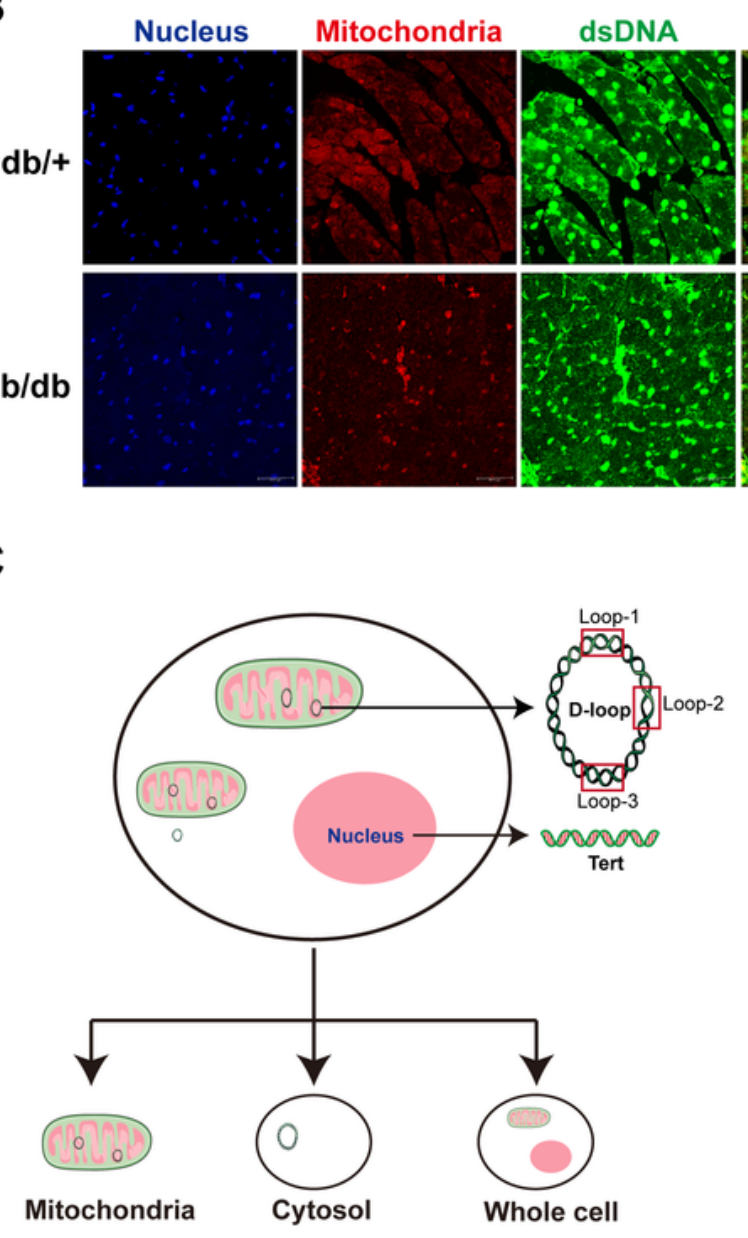

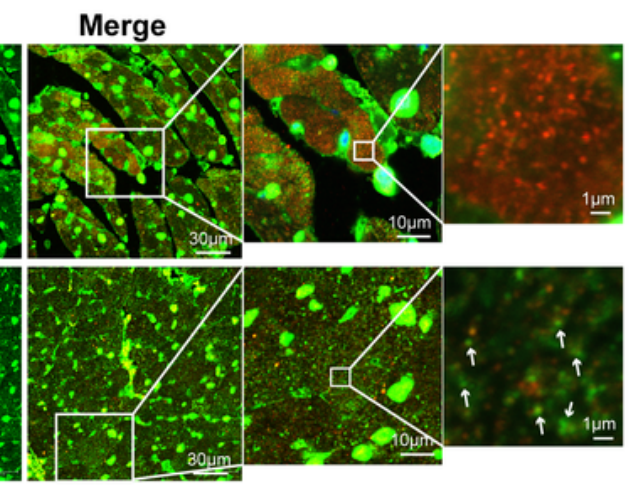

D
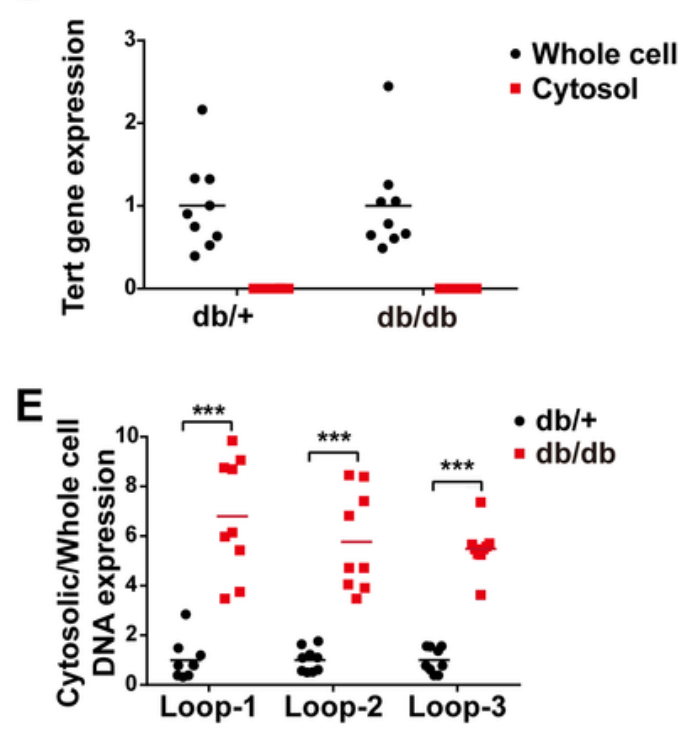

Figure 2

Mitochondria were impaired with mtDNA release in cardiomyocytes from HFD fed $\mathrm{db} / \mathrm{db}$ mice. A Representative transmission electron microscopy images of cardiomyocyte mitochondria in $\mathrm{db} /+$ mice 
and $\mathrm{db} / \mathrm{db}$ mice $(\mathrm{n}=4)$. B Detection of free dsDNA in cytoplasm of cardiomyocytes in two groups of mice by immunofluorescence double labeling, labeled mitochondria with Mitofilin (red), labeled dsDNA with anti-dsDNA (green) and labeled nucleus with DAPI (blue). ( $n=4$, the arrow indicated free dsDNA). C Schematic diagram of extraction and detection of whole cell DNA, mitochondrial DNA and cytoplasmic free DNA. D Quantitative analysis of nuclear gene Tert expression in whole cell and cytoplasm of myocardial tissue in two groups of mice $(n=9)$. E Cytosolic mtDNA content in freshly purified cardiomyocytes of $\mathrm{db} /+$ mice and $\mathrm{db} / \mathrm{db}$ mice $(\mathrm{n}=9$, $* \star * P 囚 0.001 \mathrm{vs} \mathrm{db} /+$ group).

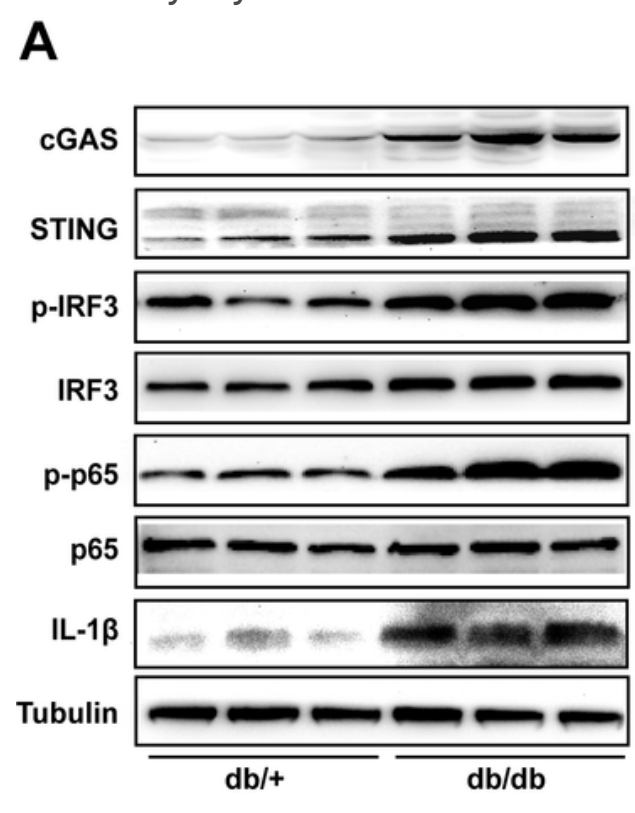

D

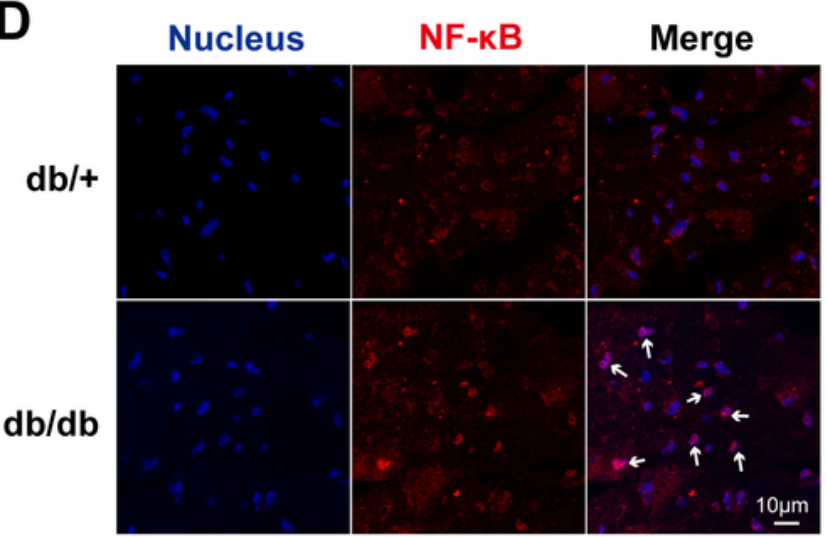

$\mathbf{E}$

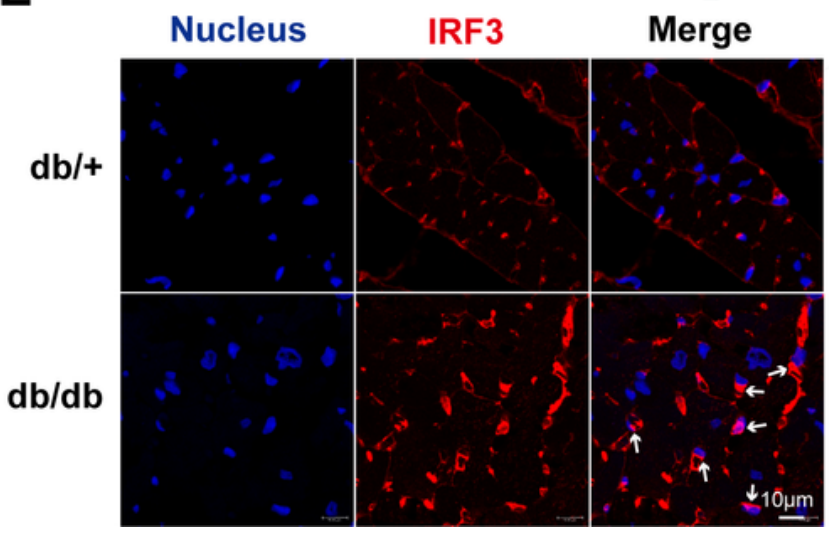

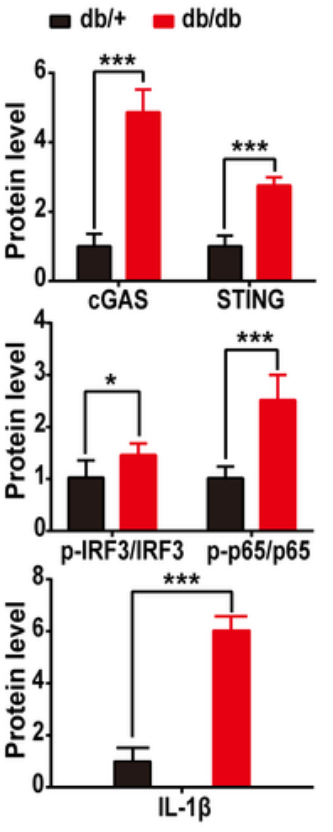

B

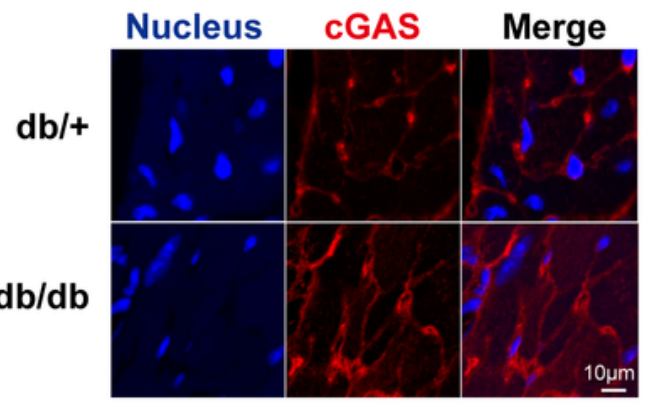

C

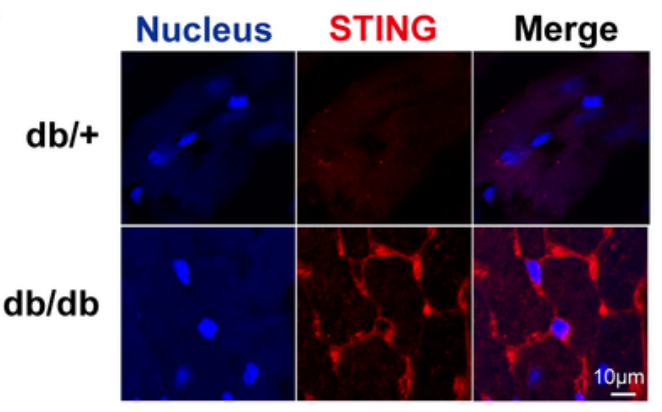

F

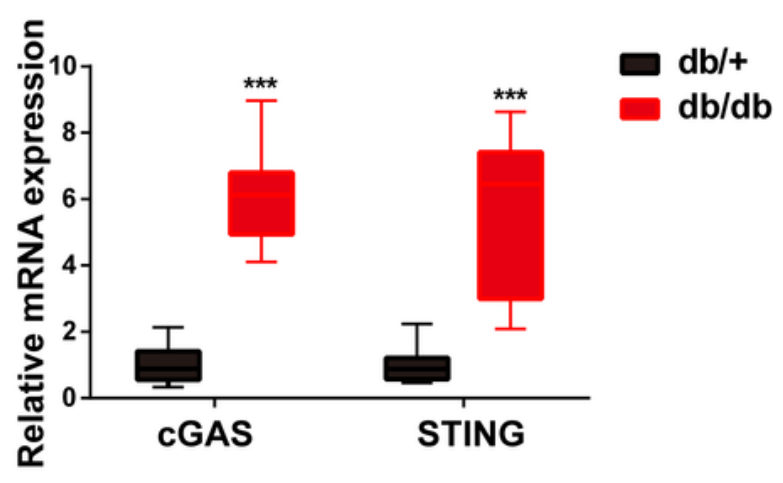

G

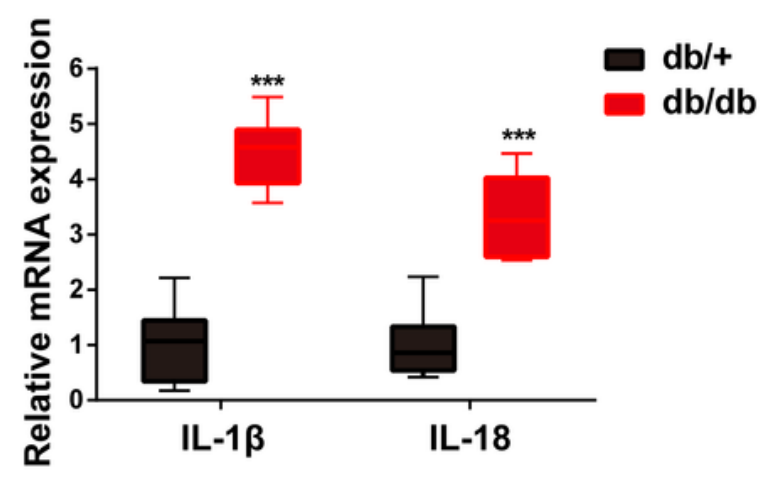




\section{Figure 3}

The cGAS-STING-IRF3/NF-KB pathway was activated in hearts of HFD-fed $\mathrm{db} / \mathrm{db}$ mice. A The protein levels of cGAS, STING, p-IRF3/IRF3, p-p65/p65 and IL-1 $\beta$ in mouse myocardium of each group ( $n=6$, *P区 $0.05,{ }^{\star * *} \mathrm{P} \otimes 0.001 \mathrm{vs} \mathrm{db} /+$ group). B-E Representative images of immunoflurescence of cGAS, STING, NF$\mathrm{KB}$ and IRF3 in mouse heart of each group. The arrow indicated the activated NF-KB and IRF3 $(n=4)$. F-G Relative mRNA level of cGAS, STING, IL-1 $\beta$ and IL-18 in mouse myocardium of each group $(n=6, * *$ P区 $0.001 \mathrm{vs} \mathrm{db/+} \mathrm{group).}$

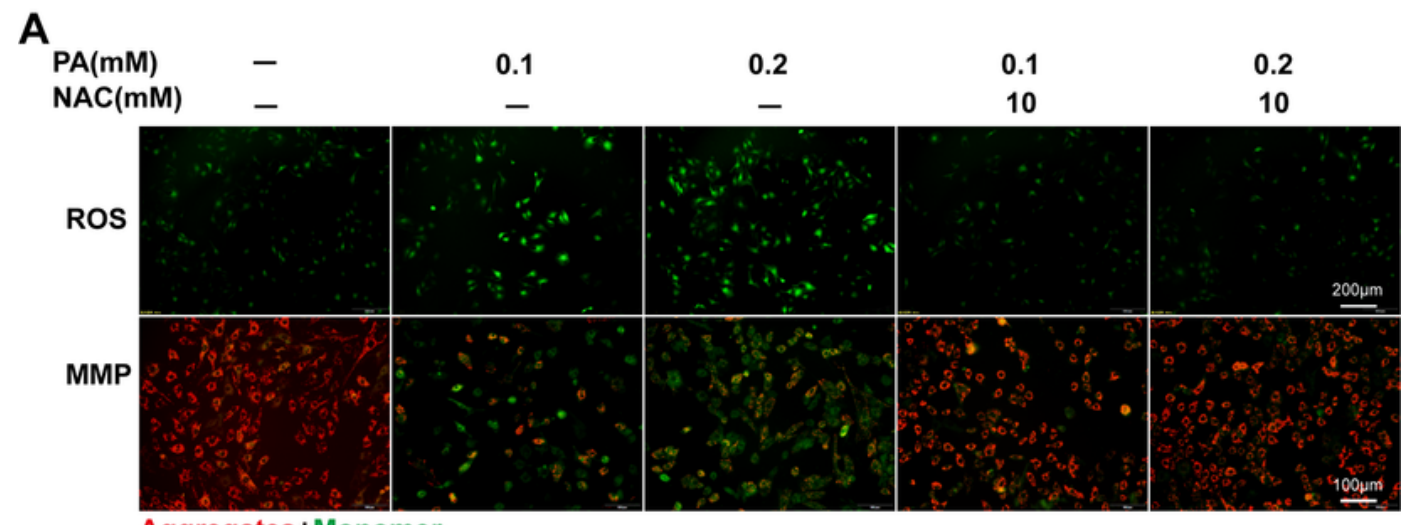

B

PA

(mM)

Ctrl

Aggregates+Monomer

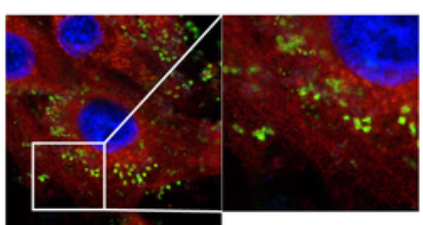

0.05

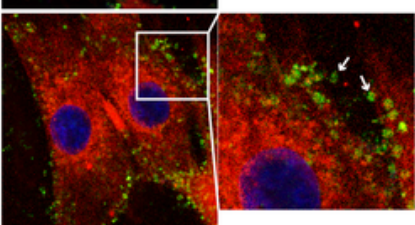

0.1

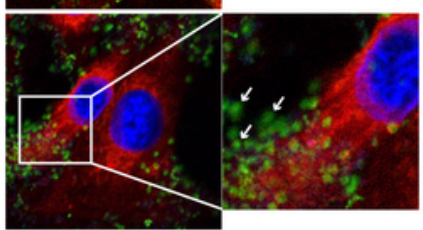

0.2

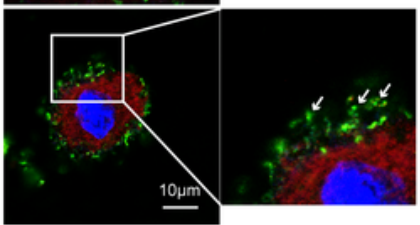

Nucleus+Mitochondria+dsDNA
C
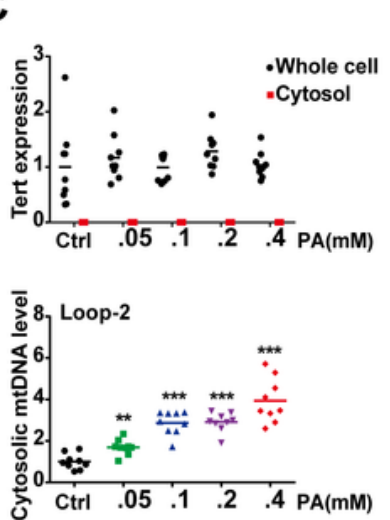
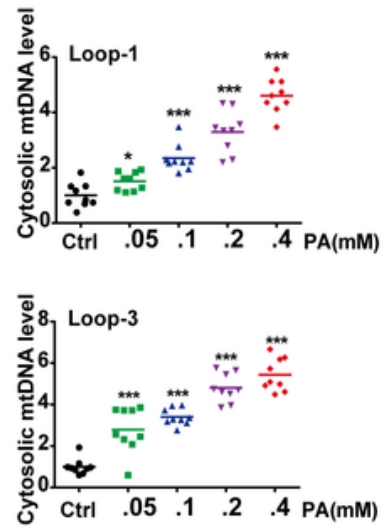

D

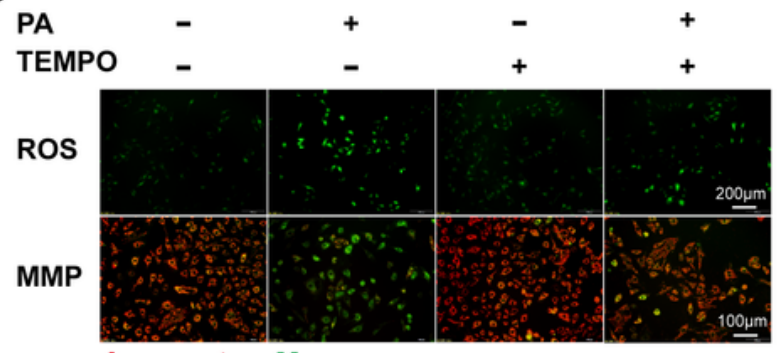

Aggregates+Monomer

E

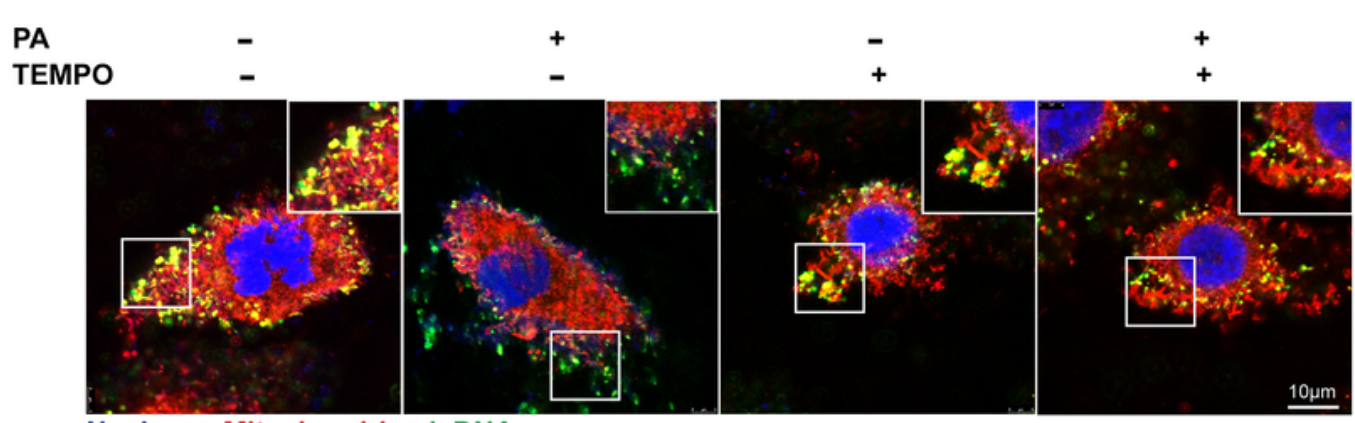




\section{Figure 4}

PA-induced mitochondrial ROS led to mitochondrial damage and mtDNA release in H9C2 cells. A ROS accumulation and the mitochondrial membrane potential (MMP) in $\mathrm{H} 9 \mathrm{C} 2$ cells treated with PA for 24 hours. ROS level was measured by DCFH-DA fluorescence and MMP was detected by JC-1 staining ( $\mathrm{n}=4$ cultures per group). B Confocal fluorescence microscopic images of $\mathrm{H} 9 \mathrm{C} 2$ cells after 24h PA-treated, labeling dsDNA with anti-dsDNA (green), mitochondria with mito-tracker (red) and nuclei with DAPI (blue) $(\mathrm{n}=5$ cultures per group). The arrow indicated free dsDNA. C Nuclear-encoded Tert gene expression in whole-cell and cytosolic extracts, and cytosolic mtDNA content from PA-treated H9C2 cells ( $n=9$ cultures per group). D ROS accumulation and MMP in PA-treated H9C2 cells $(n=4$ cultures per group, PA: $0.2 \mathrm{mM}$ for $2 \mathrm{~h}$, mito-TEMPO: $0.1 \mathrm{mM}$ for $2 \mathrm{~h}$ ). E Confocal fluorescence microscopic images of H9C2 cells after PAtreated, labeling dsDNA with anti-dsDNA (green), mitochondria with anti-mitofilin (red) and nuclei with DAPI (blue). Yellow staining indicates overlapping distribution of dsDNA and mitochondria in the merged images, green staining indicates dsDNA dissociated from mitochondria ( $n=4$ cultures per group, PA: $0.2 \mathrm{mM}$ for $2 \mathrm{~h}$, mito-TEMPO: $0.1 \mathrm{mM}$ for $2 \mathrm{~h}$ ). 
A
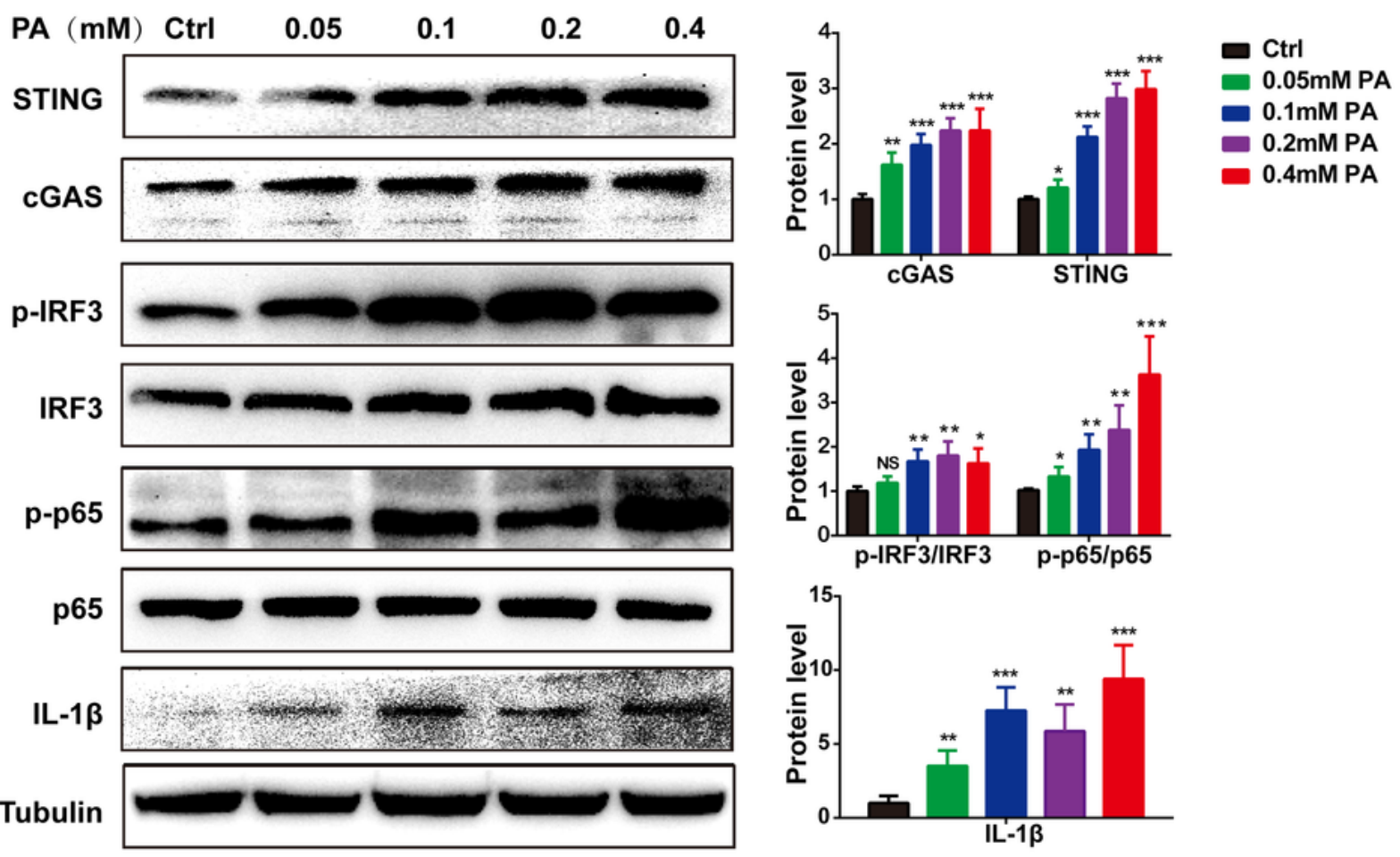

B
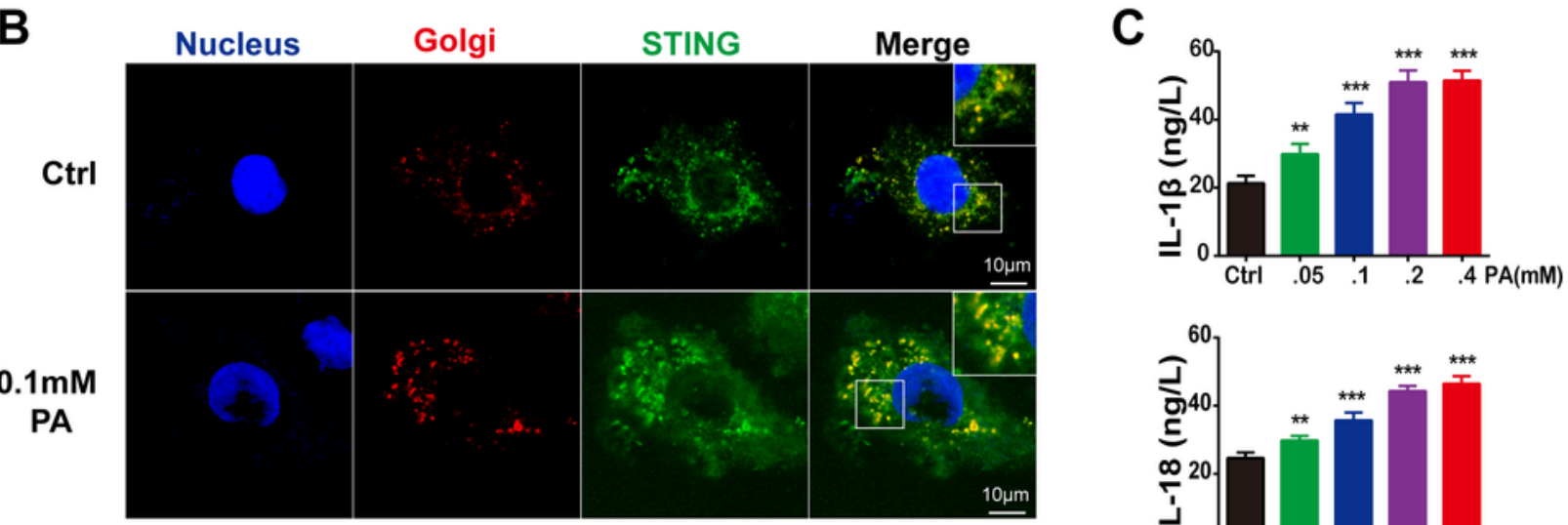

\section{$0.1 \mathrm{mM}$}

PA
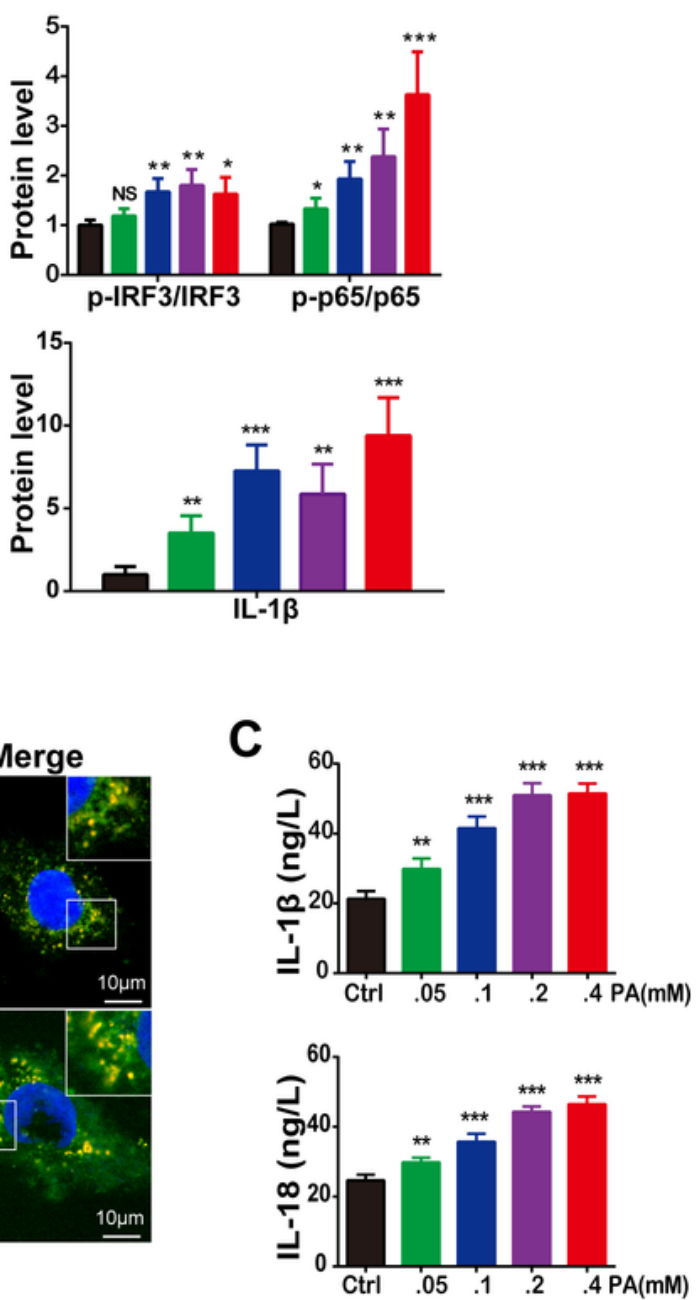

D
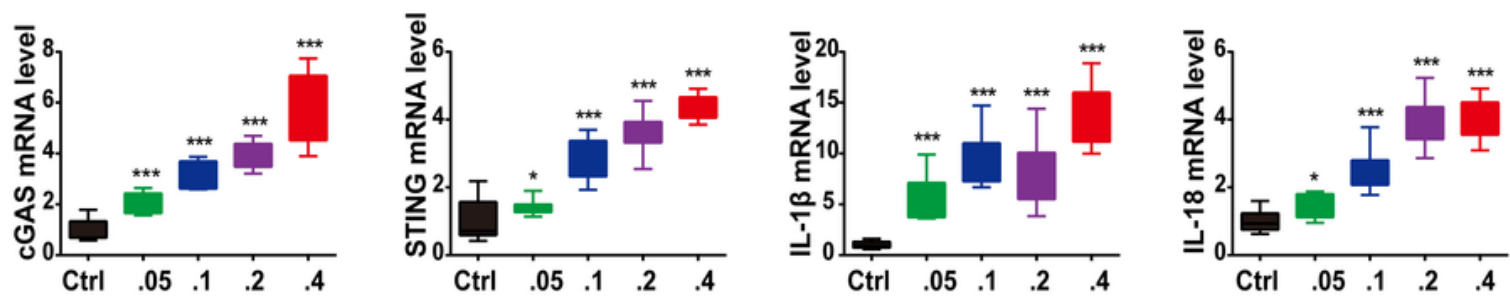

\section{Figure 5}

PA-induced activation of the cGAS-STING pathway in H9C2 cells. A The protein levels of cGAS, STING, $p$ -

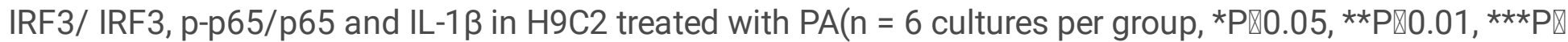
0.001 vs ctrl group, NS no significance). B Confocal fluorescence microscopic images of H9C2 cells after treated with $0.1 \mathrm{mM}$ PA for 24h, labeling STING with anti-STING (green), golgi with GM130 (red) and nuclei with DAPI (blue). Yellow staining indicates overlapping distribution of STING and golgi in the merged 
images, green staining indicates STING dissociated from golgi ( $n=5$ cultures per group). C The concentration of IL-1 $\beta$ and IL-18 in the supernatant after $\mathrm{H} 9 \mathrm{C} 2$ cells were stimulated by PA with concentration gradient for 24 hours ( $n=6$ cultures per group, ${ }^{\star * P} \otimes 0.01,{ }^{\star} * * P \otimes 0.001$ vs ctrl group). $D$ Relative mRNA level of cGAS, STING, inflammatory genes IL-1 $\beta$ and IL-18 in H9C2 cells treated with PA for

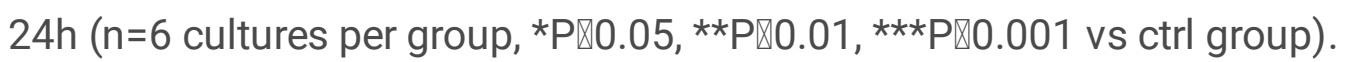

A

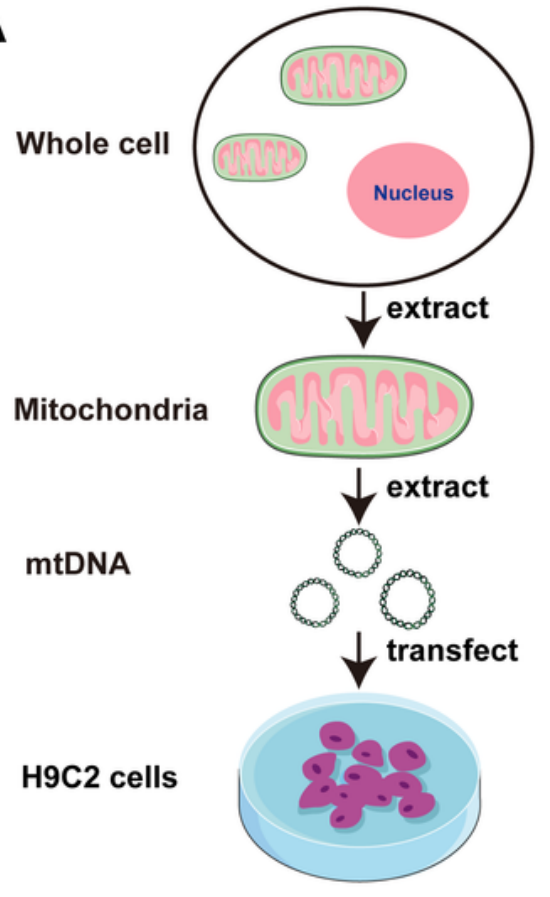

B
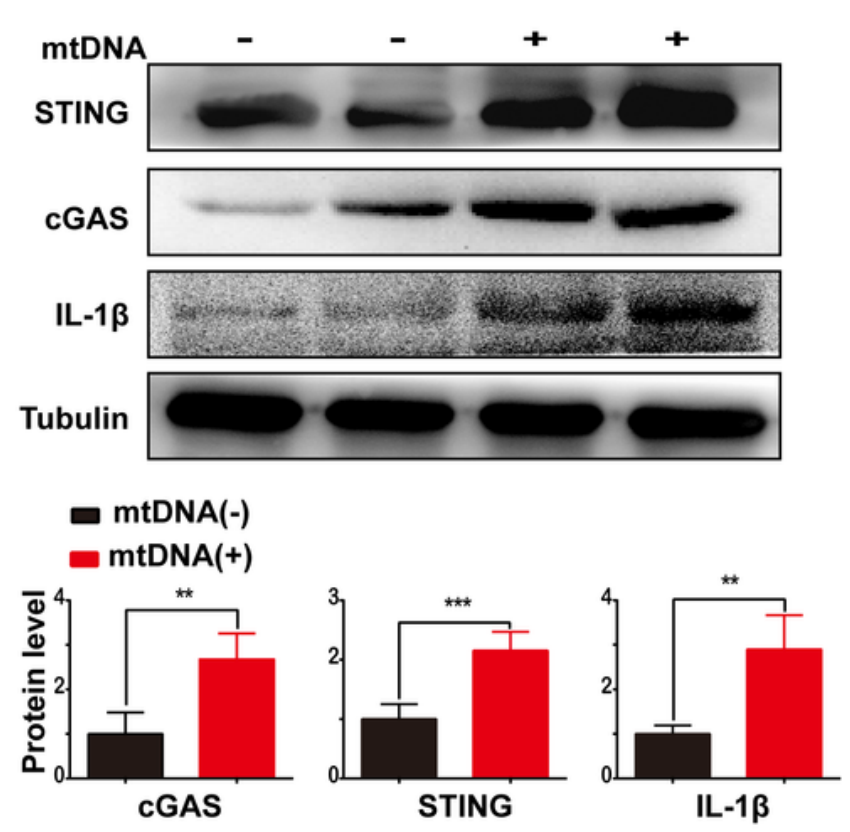

C

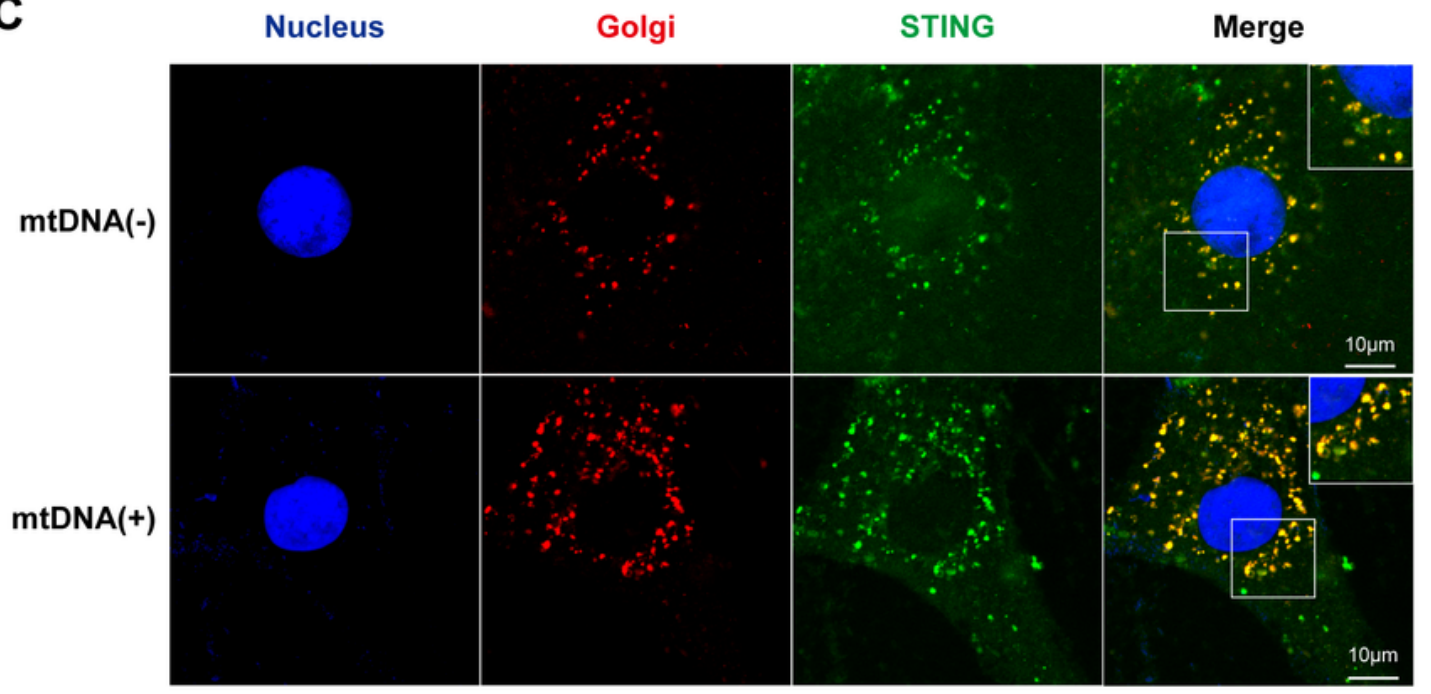

D
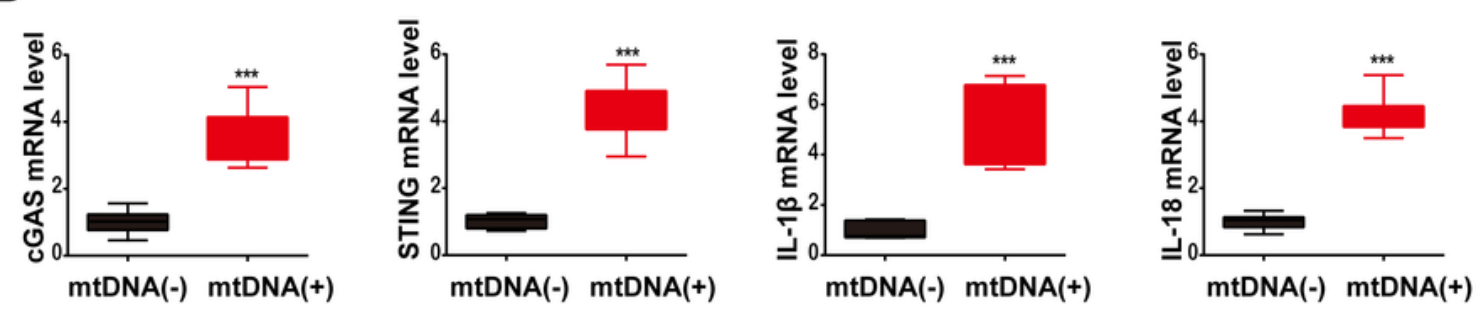

Figure 6 
Extracted mtDNA is sufficient to activate cGAS-STING signaling in $\mathrm{H} 9 \mathrm{C} 2$ cells. A Flow chart of mitochondrial DNA extraction and transfection. B The protein levels of cGAS, STING and IL-1 $\beta$ after mtDNA transfection of $\mathrm{H} 9 \mathrm{C} 2$ cells $\left(\mathrm{n}=6\right.$ cultures per group, mtDNA $3 \mu \mathrm{g}$ for $24 \mathrm{~h},{ }^{\star *} \mathrm{P} \otimes 0.01$, ${ }^{\star \star *} \mathrm{P} \otimes 0.001$ vs mtDNA(-) group). C Confocal fluorescence microscopic images of $\mathrm{H} 9 \mathrm{C} 2$ cells after transfected with $3 \mu \mathrm{g}$ mtDNA for 24h, labeling STING with anti-STING (green), golgi with GM130 (red) and nuclei with DAPI (blue). Yellow staining indicates overlapping distribution of STING and golgi in the merged images, green staining indicates STING dissociated from golgi ( $n=4$ cultures per group). D Relative mRNA level of cGAS, STING, inflammatory genes IL-1 $\beta$ and IL-18 after mtDNA transfection of H9C2 cells $(n=6$, ***P区 0.001 vs mtDNA(-) group). 
A

\begin{tabular}{|llll}
\multicolumn{2}{c}{ NC siRNA } & & \multicolumn{2}{c}{ STING siRNA } \\
\hline PBS & PA & PBS & PA \\
\hline & & & \\
\hline
\end{tabular}

STING

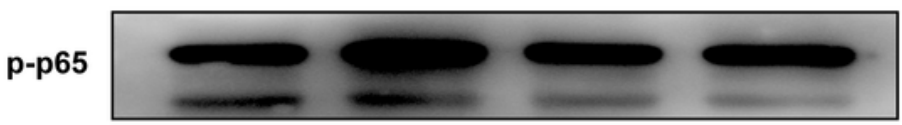

p65

IL-1 $\beta$

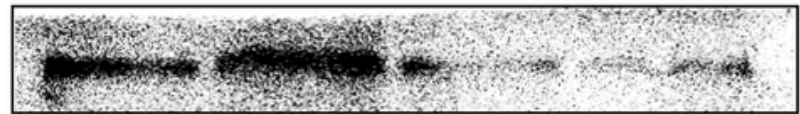

Tubulin
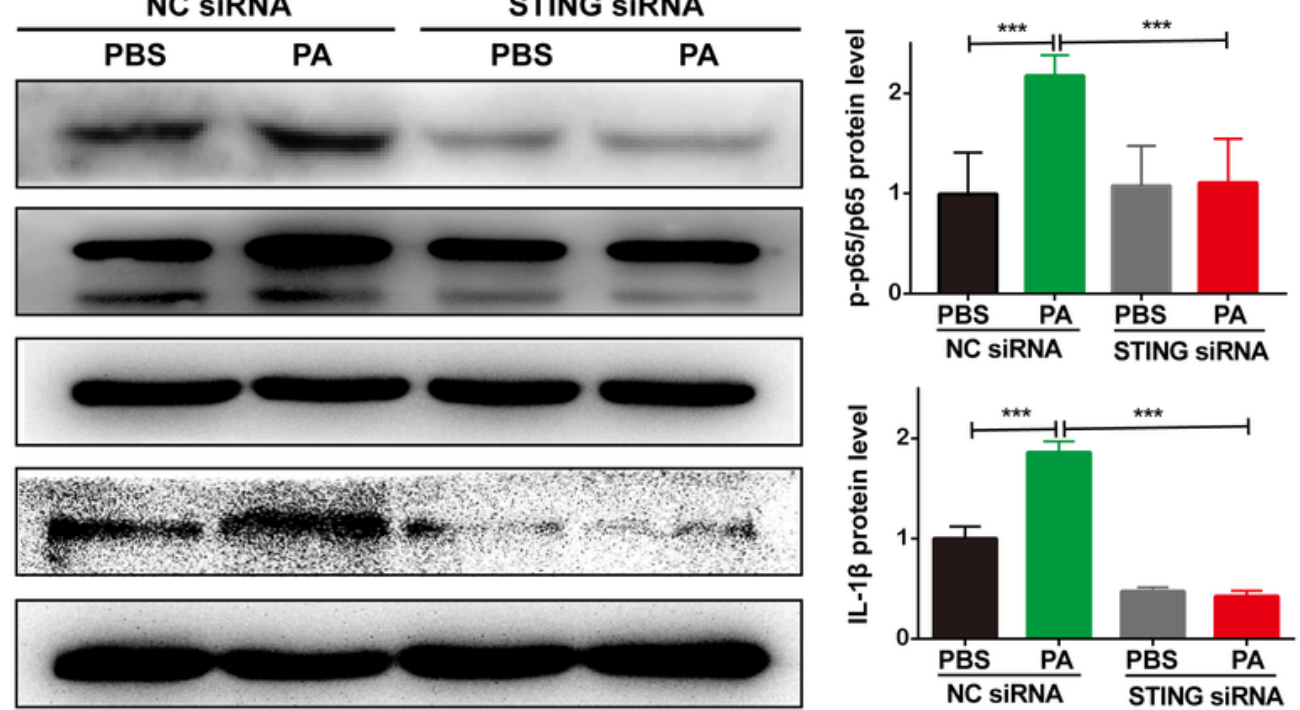

B
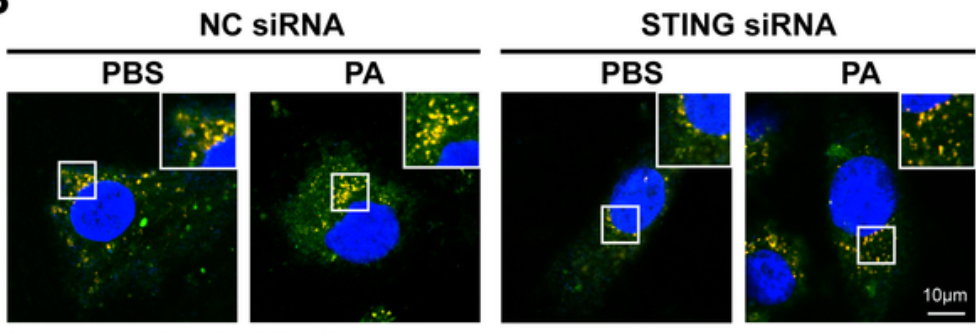

Nucleus+Golgi+STING

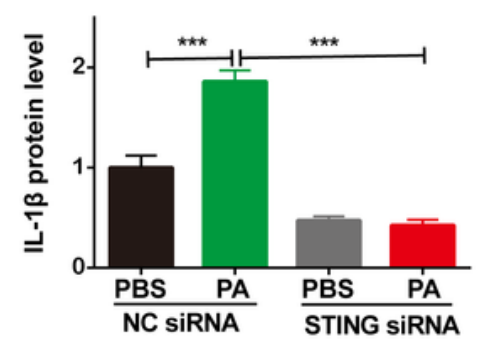

C

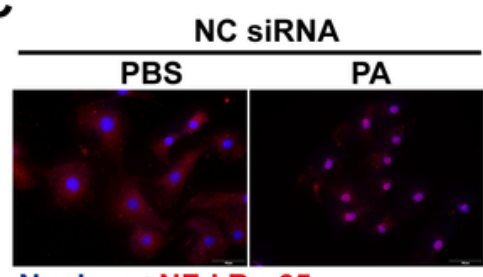

STING siRNA

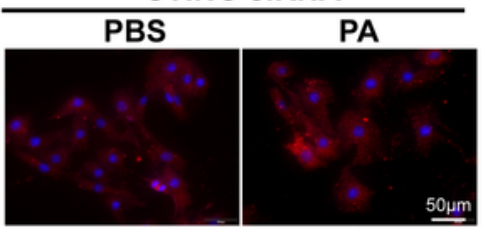

Nucleus+NF-kB p65

D

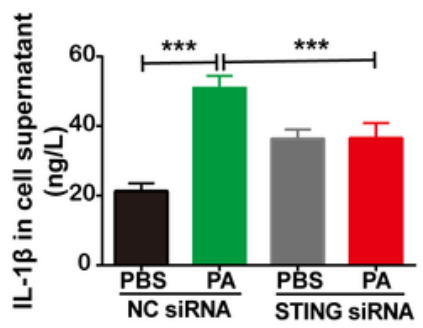

E

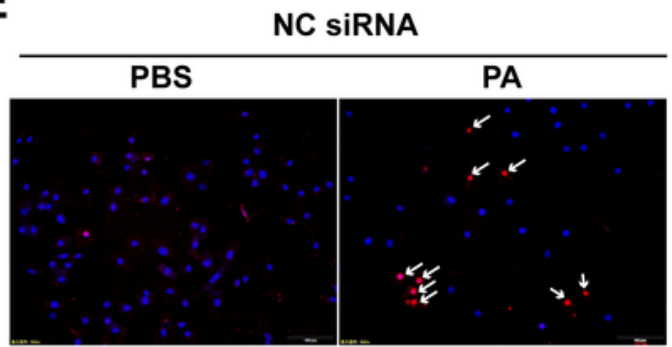

DAPI Tunel

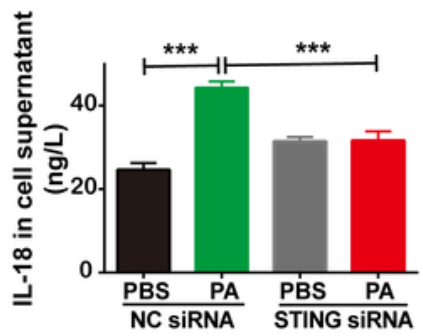

Figure 7

Knockdown of STING blocked the PA-induced inflammation and apoptosis in $\mathrm{H} 9 \mathrm{C} 2$ cells. A The protein levels of STING, p-p65/p65 and IL-1 $\beta$ in PA-treated H9C2 cells after STING knockdown by siRNA $(n=6$ cultures per group, ${ }^{\star \star *} \mathrm{P} \otimes 0.001$ vs indicated group). B Confocal fluorescence microscopic images of PAtreated $\mathrm{H} 9 \mathrm{C} 2$ cells after STING knockdown by siRNA, labeling STING with anti-STING (green), golgi with GM130 (red) and nuclei with DAPI (blue). Yellow staining indicates overlapping distribution of STING and 
golgi in the merged images, green staining indicates STING dissociated from golgi ( $n=4$ cultures per group). C Representative images of immunoflurescence of NF-KB in H9C2 cells transfected by STING siRNA ( $n=4$ cultures per group vs indicated group). D The concentration of IL-1 $\beta$ and IL-18 in the supernatant of $\mathrm{H} 9 \mathrm{C} 2$ cells transfected by STING siRNA ( $n=6$ cultures per group, ${ }^{\star \star *} \mathrm{P} \otimes 0.001$ vs indicated group). E Representative apoptosis images of $\mathrm{H} 9 \mathrm{C} 2$ cells treated by PA after NC siRNA or STING siRNA transfected, reflected by TUNEL staining. The arrow indicated apoptotic cell ( $n=6$ cultures per group).

A

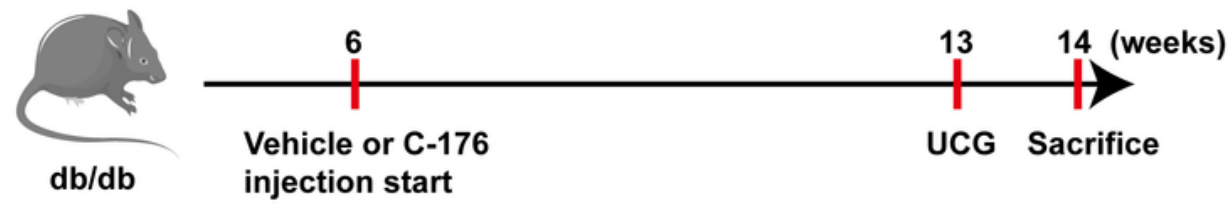

B
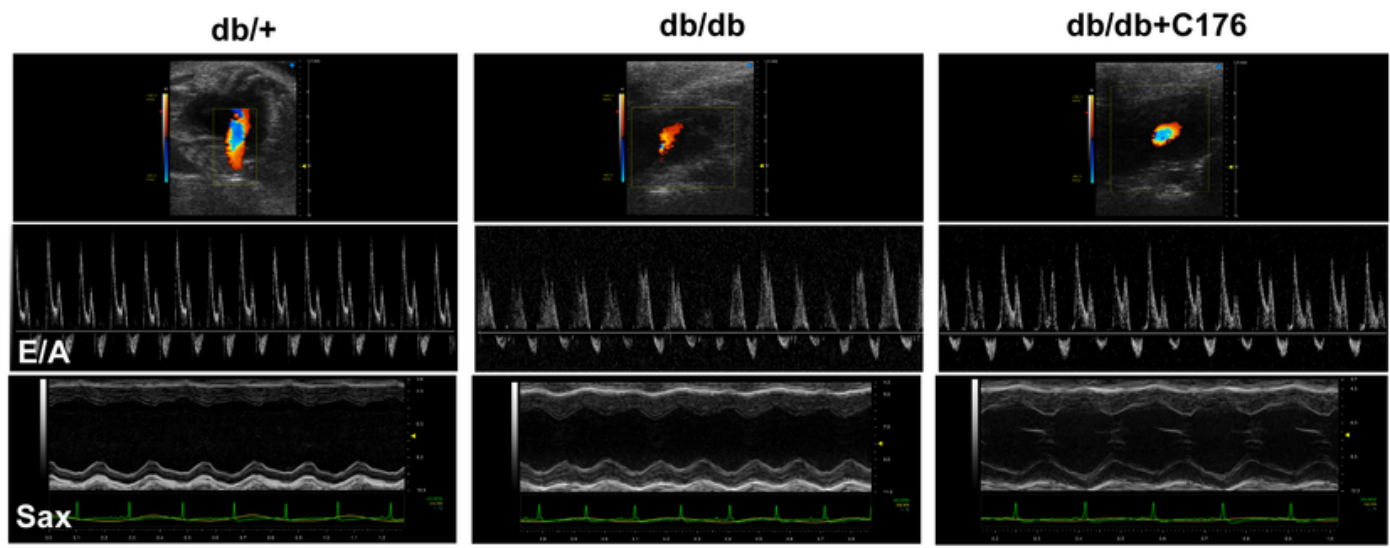

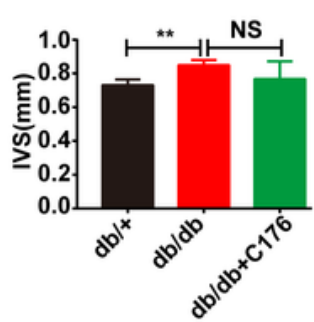

C
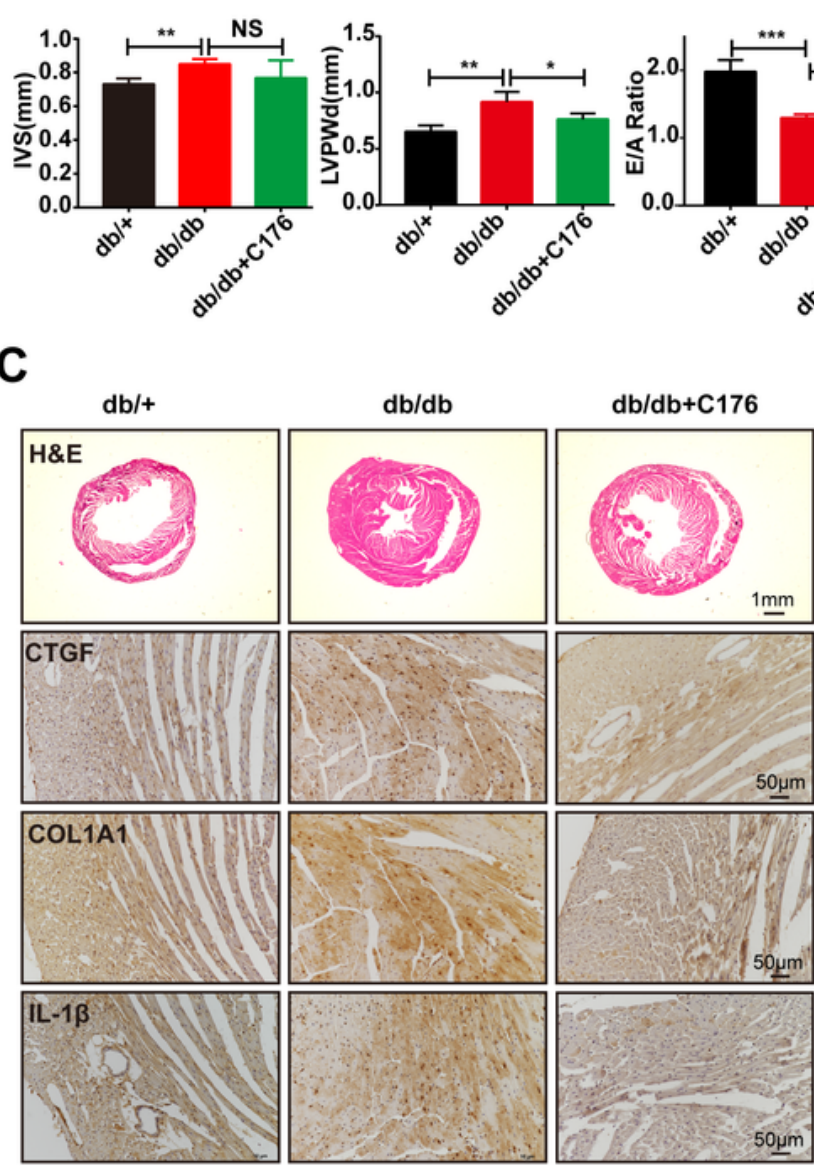
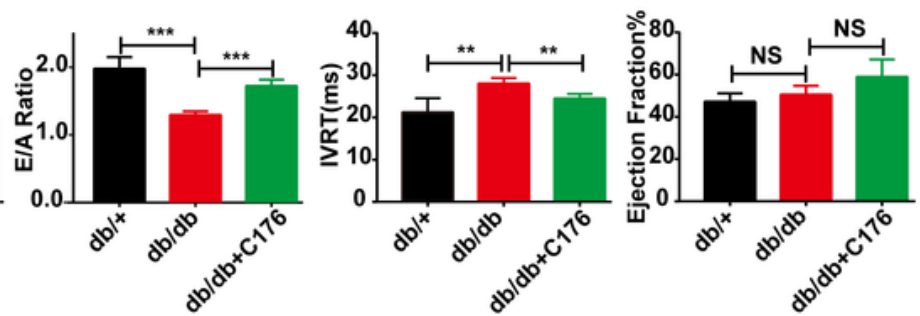

D
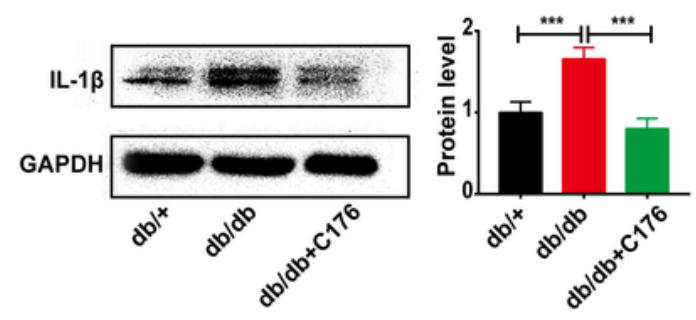

E
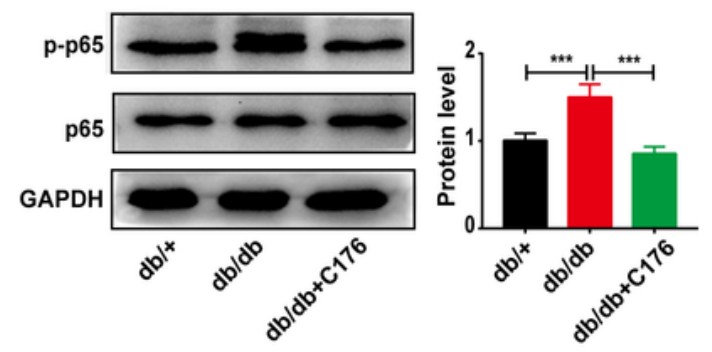

Figure 8 
Inhibition of STING ameliorated diabetic cardiomyopathy in HFD fed $\mathrm{db} / \mathrm{db}$ mice. A Flow chart of mouse feeding (C176, STING inhibitor, $750 \mathrm{nmol}$ per mouse daily in $200 \mu \mathrm{l}$ corn oil, intraperitoneal injection. UCG, Ultrasound Cardiogram). B Representative echocardiographic images of each group. IVS (interventricular septal thickness), LVPW (posterior wall thickness of left ventricle), EF\% (ejection fraction), E/A ratio and IVRT (isovolumic relaxation time) $\left(\mathrm{n}=4,{ }^{*} \mathrm{P} \otimes 0.05, * * \mathrm{P} \otimes 0.01, * \star * \mathrm{P} \otimes 0.001, \mathrm{NS}\right.$ no significance). C

Representative mouse myocardial images of the morphological analysis by H\&E staining and fibrosis analysis labeled with CTGF, COL1A1 by immunohistochemistry staining $(n=4)$. D The protein levels of IL$1 \beta$ in mouse myocardium $(n=4, * \star * P \otimes 0.001)$. E The protein levels of $p-p 65 / p 65$ in mouse myocardium $(n=4, * \star * P \otimes 0.001)$. 


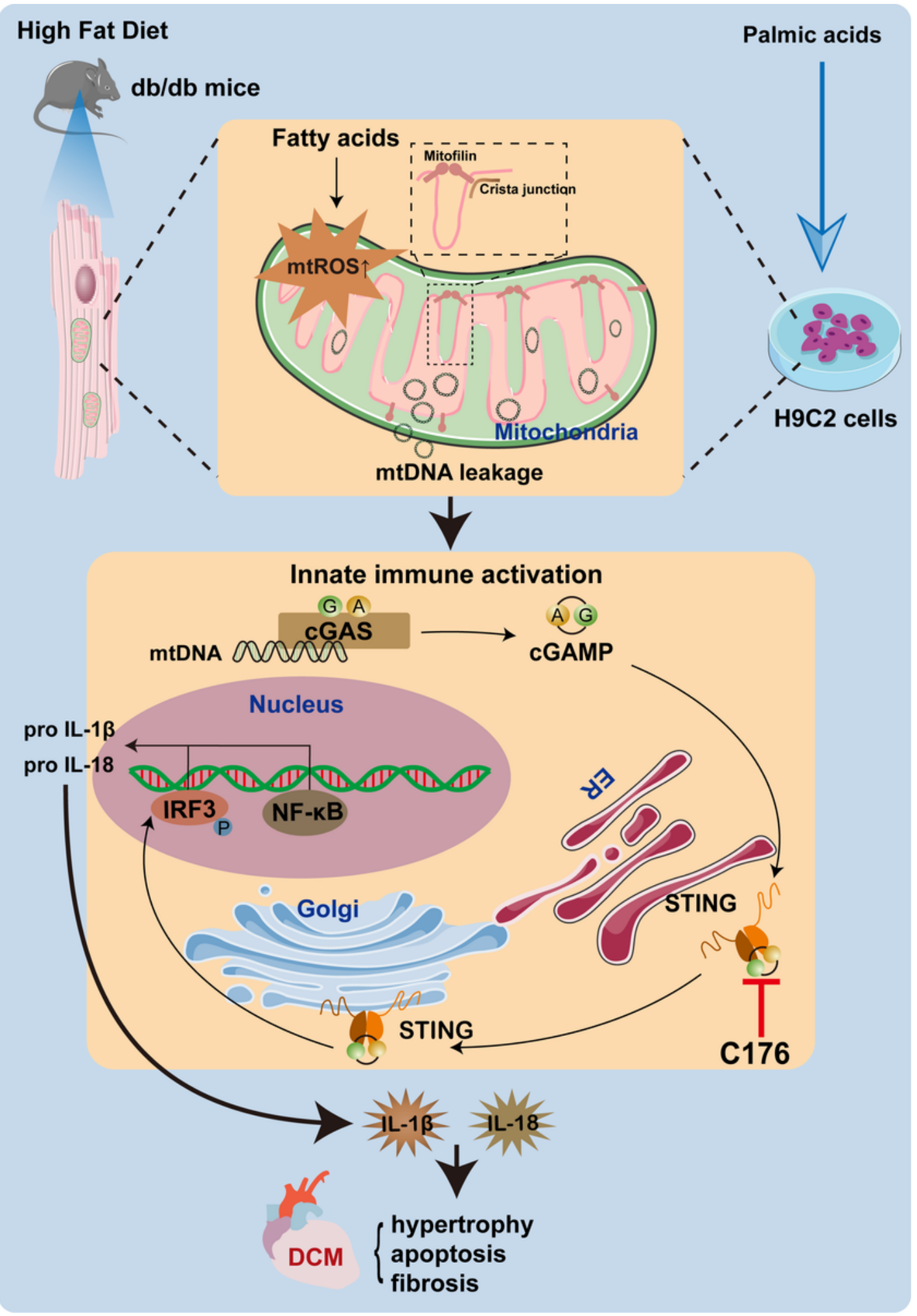

Figure 9

A diagrammatic sketch illustrating the mechanism by which mtDNA-cGAS-STING contributes to inflammation, apoptosis, hypertrophy and fibrosis of cardiomyocytes in obesity related diabetic cardiomyopathy. 